$\begin{array}{ll}\text { Research Square } & \begin{array}{l}\text { Preprints are preliminary reports that have not undergone peer review. } \\ \text { They should not be considered conclusive, used to inform clinical practice, } \\ \text { or referenced by the media as validated information. }\end{array}\end{array}$

\title{
Short-term Effects of a Three-week Inpatient Post-COVID-19 Pulmonary Rehabilitation Program - a Prospective Observational Study
}

Markus C. Hayden ( $\square$ markus.hayden@klinik-bad-reichenhall.de)

Klinik Bad Reichenhall https://orcid.org/0000-0003-3934-484X

Matthias Limbach

Klinik Bad Reichenhall

Michael Schuler

Hochschule für Gesundheit Bochum: Hochschule fur Gesundheit

Steffen Merkl

Klinik Bad Reichenhall

Gabriele Schwarzl

Klinik Bad Reichenhall

Katalin Jakab

Klinik Bad Reichenhall

Dennis Nowak

Klinikum der Universität München Institut und Poliklinik für Arbeits- Sozial- und Umweltmedizin: Klinikum der Universitat Munchen Institut und Poliklinik fur Arbeits- Sozial- und Umweltmedizin

Konrad Schultz

Klinik Bad Reichenhall

Research Article

Keywords: COVID-19, pulmonary rehabilitation (PR), dyspnea, modified Medical Research Council (mMRC), symptomatic

Posted Date: June 8th, 2021

DOI: https://doi.org/10.21203/rs.3.rs-578230/v1

License: (c) (1) This work is licensed under a Creative Commons Attribution 4.0 International License. Read Full License 


\section{Abstract}

Background:

The clinical course of coronavirus disease 2019 (COVID-19) varies distinctly. Particularly after severe or critical courses, i.e., after hospitalization, clinical manifestations frequently persist after the acute phase. However, symptoms may also persist after initially milder courses that can be treated in an outpatient setting. For patients who remain symptomatic after COVID-19, pulmonary rehabilitation (PR) is recommended. However, only few studies investigated the effectiveness of PR, especially considering different disease courses. The main objective was to evaluate the feasibility, safety, and efficacy of post-COVID-19 PR.

Methods:

A total of 120 post-COVID-19 patients who were referred to the Bad Reichenhall Clinic between April 2020 and January 2021 were asked to participate in this prospective observational study. PR was tailored to each patient's individual needs and was based on the current recommendations. The primary outcome dyspnea was assessed with numerical rating scales and the modified Medical Research Council (mMRC) dyspnea scale. Secondary outcomes included other symptoms such as cough and sputum; physical capacity; lung function; fatigue; quality of life (QoL); depression; and anxiety. Furthermore, patients rated the overall effectiveness of PR and their subjective change in health status.

Results:

A total of 108 patients (mean age $55.6 \pm 10.1$ years, $45.4 \%$ female) were included and were assigned to 3 groups depending on the referral mode (A: severe acute; B: severe after interval, C: mild after interval). At the end of PR, we detected improvements in the intensity of exertional dyspnea, physical capacity, QoL, fatigue, and depression in the overall group, with large effect sizes (Cohen's $d>0.8$ ). Moderate effect sizes $(0.5 \leq d<0.8)$ were found for resting dyspnea and the mMRC-dyspnea scale. Cohen's $d>0.4$ was found for vital capacity, forced expiratory volume in one second, partial oxygen pressure, and anxiety. Significant but rather small effect sizes $(0.2<d<0.4)$ were found for cough, sputum, pain, and other lung function parameters.

Conclusions:

PR is feasible, safe, and effective after acute COVID-19, which was true for all 3 groups analyzed, with a trend toward greater efficacy after severe courses of COVID-19 and an earlier start of PR after acute COVID-19. Therefore, all post-COVID-19 patients who remain symptomatic should be offered PR.

Trial registration: German Clinical Trials Register, DRKS00023180. Registered 01 September 2020 - Retrospectively registered, https://www.drks.de/drks_web/navigate.do?navigationld=trial.HTML\&TRIAL_ID=DRKS00023180

\section{Background}

The clinical course of SARS-CoV-2 infection is highly variable, ranging from asymptomatic courses [1] to various degrees of severity of coronavirus disease (COVID-19). In addition to mild and moderate diseases, which can mostly be treated on an outpatient basis, severe or critical COVID-19 courses [2] require hospital treatment and even admission to an intensive care unit with ventilation. In addition to initial symptoms mostly related to the respiratory organs, ranging from fever, cough, and dyspnea to respiratory failure requiring oxygen therapy or ventilation, acute COVID-19 is associated with a variety of extrapulmonary organ involvement and symptoms [3-5]. Particularly after severe or critical courses, i.e., after hospitalization, clinical manifestations often (up to $76 \%$ [6-9]) do not regress after the acute phase of the disease, so many patients still suffer both physically and psychologically from persistent impairments after an acute infection $[6-8,10]$. In addition, although it is less common, even after initially milder courses that could be treated on an outpatient basis, symptoms can persist over time [11-14]. The term postacute COVID-19 syndrome (PACS) has been proposed to describe the presence of persistent signs and symptoms beyond 4 weeks after the onset of symptoms in patients with current negative polymerase chain reaction (PCR) test results [10]. If the signs and symptoms persist for more than 3 months after the onset of the disease, the condition is labeled "post-COVID syndrome" according to the National Institute for Health and Care Excellence (NICE) [15]. For patients who remain symptomatic after acute COVID-19, rehabilitation is recommended [7, 16-18]. However, little is known about the efficacy of pulmonary rehabilitation (PR) directly after or a certain time interval after acute COVID-19 [19, 20]. In addition, the optimal timing of PR after COVID-19 has not been clarified. For example, the "COVID-19: Interim Guidance on Rehabilitation in the Hospital and Post-Hospital Phase from a European Respiratory Society and American Thoracic Society-coordinated International Task Force" [16] suggests that COVID-19 survivors with a need for rehabilitative interventions at 6 to 8 weeks following hospital discharge should participate in a comprehensive rehabilitation program, whereas in Germany, comprehensive inpatient PR often takes place directly after hospital discharge in the form of a direct transfer from the hospital to a rehabilitation clinic. In Germany, PR is usually conducted as a 3-week inpatient intervention in specialized rehabilitation centers and is a part of the social insurance system. The prerequisite for the approval of PR by German insurance providers is a persistent physical, psychological, and/or social consequence of the illness. These persistent consequences include, for example, persistent physical or psychological sequelae of the disease or a threat to the ability to work or to the ability to care for oneself. There are three ways post-COVID-19 patients may be referred to a rehabilitation clinic. First, they may be transferred directly from the hospital; second, they can be discharged into home care first and admitted to the rehabilitation clinic after either a few days or after a longer period (a few weeks to several months); and third, they can be admitted to the rehabilitation clinic after having exclusively been treated in an outpatient setting.

To reduce the research gap regarding the efficacy, usefulness, and timing of post-COVID-19 PR, the aims of this study were to assess the pre-post changes in dyspnea (primary outcome) and other clinically relevant outcomes (secondary outcomes) after PR as well as to evaluate the feasibility and safety of PR. Furthermore, we aimed to determine predictors of a successful treatment outcome, as defined by a significant reduction in dyspnea.

\section{Design And Methods}




\section{Setting and patients}

This prospective observational study was conducted at the Bad Reichenhall Clinic (Bad Reichenhall, Germany), one of the largest inpatient PR centers for adult patients in Germany. The study protocol was approved by the ethics committee of the medical faculty of the Ludwig-Maximilians-University Munich, Germany (No. 20-326) and is registered in the German register of clinical studies (DRKS00023180). All patients who were referred to the Bad Reichenhall Clinic due to postacute COVID-19 between April 28th, 2020 and January 08th, 2021 were asked to participate in the study on the first day of PR. Exclusion criteria were lack of language or cognitive abilities to fill out questionnaires. All participants provided written informed consent.

\section{Intervention}

The three-week inpatient PR program was performed in accordance with the recommendations of the German Respiratory Society (Deutsche Gesellschaft für Pneumologie) for PR in patients with COVID-19 [13]. The program was tailored to each patient's individual needs and included the following rehabilitative therapy components $(\mathrm{O}=$ obligatory for all participants, except those with individual contraindications, $\mathrm{F}=$ facultative if needed):

1. Physical training (0) was based on the recommended exercise program modalities by the German Respiratory Society [17] and consisted of two obligatory main components: a) endurance training was scheduled as 3-4 supervised units per week for 30-60 minutes each time if this was tolerated by the patient; otherwise, the duration and frequency were adjusted individually. Exercise intensity was controlled by a pulse oximeter, modified 0-10 BORG scale [21], and heart rate. b) Strength training was scheduled for 2-3 supervised sessions per week of 45-60 minutes each if tolerated; otherwise, the duration and frequency were adjusted individually. Whole-body vibration training $(F)$ was performed 7 times per week and was used only if there was no clinical evidence of thromboembolic complications and if no elevated D-dimer levels were identified. Inspiratory muscle training (F) was provided for patients with inspiratory muscle weakness and was scheduled for 7 sessions per week for 21 minutes each, of which 1 session per week was supervised.

2. Respiratory physiotherapy (0) consisted of 2 units of group respiratory physiotherapy per week for 45 minutes each session. If necessary, patients also received the following optional components: (F) a) individual breathing training by physiotherapists, (b) physiotherapy seminar on coughing techniques, and (c) mucolytic inhalation therapies (e.g., $\mathrm{NaCl}$ inhalation).

3. General physiotherapy (F), e.g., physiotherapeutic pain management, hand and foot function training, gait training, or fascia training .

4. Patient information about COVID-19 (0) in the form of a 45-minute presentation by a respiratory physician.

5. Routine medical diagnostics (0): Medical admission and discharge examination, comprehensive lung function and laboratory diagnostics, blood gas analysis, 6-minute walk test [22] with measurement of oxygen saturation, and cardiological function diagnostics (electrocardiogram obligatory, echocardiography if indicated). If necessary, further imaging examinations; psychological, psychiatric, or orthopedic examinations; or other specialist consultations were carried out.

6. Close medical supervision (0): All patients received regular medical visits, during which all therapy components, including drug therapy, were reviewed, and adjusted if necessary. Oxygen therapy at rest and especially during exertion, e.g., as part of exercise therapy, was available. Noninvasive ventilation could be continued during PR and monitored accordingly.

7. Psychosocial support, in the form of a psychologically guided self-help group on COVID-19 (0). Social counseling (F) and/or individual clinical psychological counseling (F) were offered if necessary. Neuropsychological diagnostics of cognitive performance were conducted if patients showed signs of cognitive impairment. Furthermore, relaxation seminars (F), such as progressive muscle relaxation or autogenic training, were offered.

8. Nutritional counseling $(\mathrm{F})$ was offered, for example, for patients with overweight, diabetes, or other comorbid disorders.

9. Occupational therapy $(\mathrm{F})$, e.g., prescription and consultation regarding necessary aids, memory training, or training regarding activities of daily life.

\section{Outcomes and measures}

\section{Primary outcome}

The primary outcome dyspnea was assessed based on the current dyspnea sensation and dyspnea in the last 7 days. On the one hand, we used an 11-point numeric rating scale [23] (NRS) to assess the present severity of dyspnea sensation at rest and on exertion. The scales have two dimensions (symptom intensity and symptom unpleasantness) and range from 0 ("no symptoms") to 10 ("worst imaginable symptom severity"). The minimal clinically important difference is considered to be 1 to 2 points $[24,25]$.

On the other hand, the modified Medical Research Council (mMRC) dyspnea scale [26-28] was used to rate the impact of dyspnea on daily activities over the last week on a 5-point scale, ranging from 0 ("I only get breathless with strenuous exercise") to 4 ("I am too breathless to leave the house or I am breathless when dressing"). The mMRC is considered to have low sensitivity to change, and a reduction of 1 point can be considered clinically relevant [29].

\section{Assessment of secondary outcomes and further variables Physical capacity}

The 6-minute walking distance (6MWD) was measured using a track length of $30 \mathrm{~m}$ according to the European Respiratory Society's and American Thoracic Society's technical standards [22]. The deviations from the healthy reference values were calculated according to Enright and Sherrill [30].

Page $3 / 22$ 


\section{Lung function tests, blood gases, and laboratory blood tests}

Forced expiratory volume in one second (FEV1), vital capacity (VC), residual volume (RV), total lung capacity (TLC), total specific airway resistance (sRtot), maximal inspiratory pressure (PImax), and the single-breath transfer factor of the lung for carbon monoxide (TLCO) were determined using spirometry and body plethysmography (MasterScreen Body, CareFusion, Hoechberg, Germany) and MasterScreen Diffusion System, Jaeger-Viasys, CareFusion), and respiratory muscle testing was performed using a Jaeger mouth occlusion pressure device in accordance with recommendations of the national guidelines $[31,32]$. Capillary blood gas samples to assess the partial oxygen pressure $(\mathrm{PaO} 2)$ and partial carbon dioxide pressure (PaCO2) were taken at rest while breathing ambient air (ABL 800, Radiometer, Willich, Germany). Laboratory chemistry tests, including D-dimers, brain natriuretic peptide (BNP), lactate dehydrogenase (LDH), and C-reactive protein (CRP), were performed by a certified external laboratory (SYNLAB Holding, Augsburg, Germany).

\section{Cardinal symptom}

The patients were asked to name their "most important symptom". To ease the process, we presented a list of symptoms that are frequently associated with COVID-19. Further symptoms could be specified by the patients.

\section{Cough, sputum, and pain}

Cough, sputum, and pain were assessed with 11-point NRSs, ranging from 0 ("no symptoms") to 10 ("worst imaginable symptom severity").

\section{Fatigue}

The German version of the Brief Fatigue Inventory (BFI [33]) was used to assess fatigue. This questionnaire assesses the severity of and impairment from fatigue with ten questions. The 9 subscales and the total score range from 0 to 10 , with higher values indicating higher severity/impairment. Scores of 4 to 6 correspond to "moderate fatigue", and scores of 7 to 10 correspond to "severe fatigue" [34].

\section{Quality of life}

Generic health-related quality of life (QoL) was measured using the 5-level EuroQol questionnaire (EQ-5D-5L) and the EuroQol visual analog scale (VAS) [35]. The descriptive system of the EQ-5D-5L comprises five dimensions (mobility, self-care, usual activities, pain/discomfort, and anxiety/depression). Each dimension has five levels: "no problems", "slight problems", "moderate problems", "severe problems", and "extreme problems". The VAS is an instrument to describe patients' current subjectively experienced QoL on a scale ranging from 0 ("worst possible health condition") to 100 ("best possible health condition"). The minimal clinically important difference in chronic obstructive pulmonary (COPD) patients is 8 points [36].

\section{Depression and anxiety}

The Patient Health Questionnaire-9 (PHQ-9) [37] and the General Anxiety Disorder-7 (GAD-7) [38] of the German version of the Patient Health Questionnaire [39] were used as screening tools to assess symptoms of depression and anxiety. All items are scored on a 4-point Likert scale $(0=$ "not at all", $1=$ "several days"; 2 = "more than half of the days"; 3 = "nearly all days"). In addition to using the sum of scores (ranging from 0 to 27 for the PHQ-9 and 0 to 21 for the GAD-7), we classified individuals with values of $\geq 10$ as being at risk for a clinically relevant depressive disorder (PHQ-9) or a clinically relevant anxiety disorder (GAD-7).

\section{Estimation of the overall effectiveness of rehabilitation from the patient's perspective}

At $T_{2}$, patients were asked to rate the effectiveness of the PR on an 11-point Likert scale, ranging from 0 ("the rehabilitation was ineffective for me") to 10 ("the rehabilitation was highly effective for me").

\section{Global rating of change in subjective health}

The global rating of chance in subjective health (GROC) [40] was assessed using one item that compares current subjective health with subjective health at the beginning of inpatient rehabilitation and one item that compares current subjective health to subjective health at the time before SARS-CoV-2 infection. The response scale ranges from -7 ("much worse") to 0 ("no change") to 7 ("much better"). Changes $\geq 2$ points are considered clinically relevant, and changes $\geq 4$ and $\geq 6$ points are considered moderate and strong, respectively [40, 41].

\section{Statistical analyses}

Statistical analyses were performed using IBM SPSS (V26) and $R$ (V3.6.3). For the analyses of differences between the subsamples at baseline, we used independent-samples $t$-tests for continuous parameters and chi-square tests for categorical variables. For the analyses of changes in the outcomes between $T_{1}$ and $T_{2}$, we used repeated-measures analysis of variance (RM-ANOVA). The evaluations were performed pairwise only so that a value was calculated only if both values (T1 and T2) were available. To assess within-group effect sizes, Cohen's $d$ [42] was calculated by dividing the differences in means between $\mathrm{T}_{2}$ and $T_{1}$ by the standard deviation of the change scores. Values of $0.2,0.5$, and 0.8 are considered small, moderate, and large, respectively. We calculated $95 \%$ confidence intervals for within-group mean differences as well as for Cohen's $d$.

Predictors of therapy success were calculated using linear regression analyses. For these analyses, we calculated the changes in clinically relevant variables according to $\Delta=\mathrm{M}_{\mathrm{T} 2}-\mathrm{M}_{\mathrm{T} 1}$. Intercorrelations between the deltas were calculated using Pearson correlations for parametric variables and Spearman correlations for nonparametric variables.

\section{Results}




\section{Sample}

Between April 28th, 2020, and January 08th, 2021, 120 patients were enrolled in PR due to COVID-19. One patient was excluded from the study because of cognitive impairment, five patients were excluded because of insufficient German language skills, and six patients did not agree to participate in the study. The remaining 108 patients, consisting of 49 women (mean age: $53.67 \pm 10.67$ years; mean body mass index (BMI): $30.74 \pm 6.64)$ and 59 men (mean age: $57.20 \pm$ 9.21 years; mean BMI: $29.70 \pm 5.54$ ), gave their informed consent and were enrolled. Further baseline descriptive data are displayed in Table 1. One patient dropped out because he withdrew his consent for further study participation. Two patients dropped out because of medical conditions that were not related to PR. The rest of the sample completed the rehabilitation program without complications. The mean treatment duration of PR was $26.3 \pm 5.9$ days (range: $5-42$ days).

Table 1

Patient characteristics at baseline

\begin{tabular}{|c|c|c|c|c|}
\hline & $\begin{array}{l}\text { Group A } \\
\text { Acute severe }\end{array}$ & $\begin{array}{l}\text { Group B } \\
\text { Severe after interval }\end{array}$ & $\begin{array}{l}\text { Group C } \\
\text { Mild after interval }\end{array}$ & $\begin{array}{l}\text { All } \\
\text { patients }\end{array}$ \\
\hline Referral to rehab as .... & $\begin{array}{l}\text { PR after direct transfer from } \\
\text { hospital }(n=24) \text { or no more } \\
\text { than } 4 \text { weeks after discharge } \\
(n=31)\end{array}$ & $\begin{array}{l}\text { PR until at least } 1 \text { month } \\
\text { after discharge from hospital } \\
\text { with a hospital stay of at } \\
\text { least } 3 \text { days }\end{array}$ & $\begin{array}{l}\text { PR after outpatient treatment ( } \\
=16) \text { or monitored in the } \\
\text { hospital for a maximum of one } \\
\text { night }(n=5)\end{array}$ & \\
\hline Number of patients & $\mathrm{n}=55(50.9 \%)$ & $\mathrm{n}=32(29.6 \%)$ & $\mathrm{n}=21(19.4 \%)$ & $\begin{array}{l}N=108 \\
(100 \%)\end{array}$ \\
\hline $\begin{array}{l}\text { Age } \\
\text { [mean } \pm \mathrm{SD}] \\
\text { (range) }\end{array}$ & $\begin{array}{l}57.9 \pm 10.8 \\
(33-85)\end{array}$ & $\begin{array}{l}54.0 \pm 9.9 \\
(32-80)\end{array}$ & $\begin{array}{l}52.1 \pm 6.8 \\
(39-61)\end{array}$ & $\begin{array}{l}55.6 \pm \\
10.1 \\
(32- \\
85)\end{array}$ \\
\hline $\begin{array}{l}\text { Sex } \\
{[\% \text { female }]}\end{array}$ & $\begin{array}{l}38.2 \% \\
(34 \mathrm{M} / 21 \mathrm{~F})\end{array}$ & $\begin{array}{l}34.4 \% \\
(21 \mathrm{M} / 11 \mathrm{~F})\end{array}$ & $\begin{array}{l}81.0 \% \\
(4 \mathrm{M} / 17 \mathrm{~F})\end{array}$ & $\begin{array}{l}45.4 \% \\
(59 \\
M / 49 \\
F)\end{array}$ \\
\hline $\begin{array}{l}\text { BMI } \\
{[\text { mean } \pm \mathrm{SD}]} \\
\text { (range) }\end{array}$ & $\begin{array}{l}29.9 \pm 5.7 \\
(19.3-53.3)\end{array}$ & $\begin{array}{l}31.5 \pm 6.7 \\
(21.0-48.3)\end{array}$ & $\begin{array}{l}28.9 \pm 6.1 \\
(19.9-46.0)\end{array}$ & $\begin{array}{l}30.17 \pm \\
6.12 \\
(19.3- \\
53.3)\end{array}$ \\
\hline $\begin{array}{l}\text { Length of hospitalization } \\
\text { [mean } \pm \text { SD in days] } \\
\text { (range) }\end{array}$ & $\begin{array}{l}31.5 \pm 18.7 \\
(3-101)\end{array}$ & $\begin{array}{l}19.4 \pm 11.0 \\
(3-49)\end{array}$ & $\begin{array}{l}\text { Monitored in the hospital for a } \\
\text { maximum of one night }(n=5)\end{array}$ & $\begin{array}{l}25.7 \pm \\
17.8 \\
(1- \\
101)\end{array}$ \\
\hline $\begin{array}{l}\text { \% ICU-treatment } \\
\text { [mean } \pm \text { SD in days] } \\
\text { (range) }\end{array}$ & $\begin{array}{l}70.9 \% \\
21.7 \pm 18.1 \\
(4-97)\end{array}$ & $\begin{array}{l}56.3 \% \\
14.5 \pm 8.8 \\
(5-40)\end{array}$ & - & $\begin{array}{l}52.8 \% \\
23.6 \pm \\
17.4 \\
(5-97)\end{array}$ \\
\hline $\begin{array}{l}\text { \% Invasive ventilation } \\
\text { [mean } \pm \mathrm{SD} \text { in days] } \\
\text { (range) }\end{array}$ & $\begin{array}{l}49.1 \% \\
20.0 \pm 17.7 \\
(8-87)\end{array}$ & $\begin{array}{l}37.5 \% \\
11.6 \pm 4.9 \\
(5-23)\end{array}$ & - & $\begin{array}{l}36.1 \% \\
17.6 \pm \\
15.5 \\
(5-87)\end{array}$ \\
\hline $\begin{array}{l}\text { Duration between discharge from the } \\
\text { clinic or the acute COVID-19 phase } \\
\text { undergone in an outpatient setting and } \\
\text { beginning of PR } \\
\text { [mean } \pm \text { SD in days] } \\
\text { (range) }\end{array}$ & $\begin{array}{l}10.8 \pm 11.2 \\
(0-31)\end{array}$ & $\begin{array}{l}120.6 \pm 70.2 \\
(32-270)\end{array}$ & $\begin{array}{l}142.9 \pm 55.1 \\
(35-270)\end{array}$ & $\begin{array}{l}69.0 \pm \\
75.3 \\
(0- \\
270)\end{array}$ \\
\hline $\begin{array}{l}\% \text { Oxygen therapy during acute COVID-19 } \\
\text { phase }\end{array}$ & $87.3 \%$ & $68.8 \%$ & $14.3 \%$ & $67.6 \%$ \\
\hline
\end{tabular}

The initial acute COVID-19 diagnosis was based on PCR diagnostics in all but one case, for whom the diagnosis was based on antibody detection. All patients were PCR negative upon admission to the clinic. 
Comorbidities were common: Only 2 of the 108 patients $(1.9 \%)$ had no comorbidities. A total of $55.6 \%$ of patients had at least one cardiovascular disease (see Fig. 1), most frequently hypertension (52.8\%). A total of $31.5 \%$ of patients had at least one upper or lower airway disease; among them, 2 patients (1.85\%) had COPD, and $18(16.7 \%)$ had asthma. Only 1 patient was a current smoker, $63(58.3 \%)$ had never smoked and 44 (40.7\%) were ex-smokers.

\section{Subsamples}

The German health care system provides an administrative referral path to rehabilitation. Therefore, we clustered the patients according to the mode of admission into three groups (see Table 1). The first group (group A, 'acute severe) consisted of patients who were admitted to PR either directly after hospital discharge or within the first month after discharge from the hospital. This "follow-up rehabilitation" initiated by hospital physicians while the patient is still in the hospital is an integral part of the German rehabilitation system. These patients were still heavily burdened and still in the process of recovering. The second group (group B, 'severe after interval) consisted of patients who had been hospitalized for at least 3 days but were admitted to the PR after more than one month following discharge from the hospital. These rehabilitations were usually not initiated by the hospital but rather through family physicians or specialists in private practice. The last group (group C 'mild after interval) included patients who had been treated in an outpatient setting or who had been monitored for a maximum of one night in a hospital. These rehabilitations were initiated by physicians in private practice.

Sample characteristics are presented in Table 1. Patients in group $\mathrm{C}$ were more likely to be younger $(p=0.045)$ and female $(p=0.001)$ and to require less often oxygen therapy during the acute phase $(p<0.001)$. There were no significant differences between the three subgroups regarding BMI $(p=0.265)$. Differences regarding outcome measures are displayed in the respective sections below.

\section{Cardinal symptoms at the beginning of PR}

The most prevalent symptom was dyspnea on exertion. Fifty percent of the whole sample named this symptom the most burdensome. This finding was consistent in all groups. Descriptively, however, there were differences: In group A, 56\% reported dyspnea on exertion as the most important symptom; in group B, $50 \%$ reported dyspnea on exertion as the most important symptom; and in group C, $40 \%$ reported dyspnea on exertion as the most important symptom. Dyspnea at rest, on the other hand, was reported in less than $1 \%$ of patients. Other symptoms that were frequently mentioned were sadness regarding health status $(16.7 \%)$, faintness/lack of energy $(15.7 \%)$, and pain in various parts of the body $(7.8 \%)$. Chi-square tests of independence did not find any significant difference between the three subgroups ( $p=0.298$ to $p=0.918)$.

\section{Primary outcome: dyspnea}

The quantitative description of the intensity of dyspnea, as measured by the NRS and the mMRC, is displayed in Table 2, while the NRS results concerning unpleasantness are displayed in Table S1 of the supplement. A graphical illustration of the significant improvements in clinically relevant variables is displayed in Fig. 2. 
Results for the primary outcome measures

\begin{tabular}{|c|c|c|c|c|c|c|c|}
\hline Group & $\mathrm{T}_{1}$ & & $\mathrm{~T}_{2}$ & & & & \\
\hline & $\mathrm{M}$ & SD & $\mathrm{M}$ & SD & Delta & $d$ & RM-ANOVA \\
\hline & (Median) & (Range) & (Median) & (Range) & [95\% Cl] & [95\% Cl] & $F(d f), p$ \\
\hline \multicolumn{8}{|l|}{ Dyspnea } \\
\hline \multicolumn{8}{|c|}{ NRS “How intense is your dyspnea at rest?” ( $0-10 \wedge)$} \\
\hline All patients & 1.70 & 1.82 & 1.02 & 1.49 & -0.67 & -0.41 & \multirow{8}{*}{$\begin{array}{l}\mathrm{F}_{\mathrm{T}}=13.3(1), \\
\mathrm{p}<0.001 \\
\mathrm{~F}_{\mathrm{G}}=2.4(2), \\
\mathrm{p}=0.100 \\
\mathrm{~F}_{\mathrm{G}^{*} \mathrm{~T}}=0.24(2), \\
\mathrm{p}=0.788\end{array}$} \\
\hline$N=82$ & $(1.00)$ & $(0.00-8.00)$ & $(0.00)$ & $(0.00-7.00)$ & {$[-1.03 ;-0.32]$} & {$[-0.64 ;-0.19]$} & \\
\hline$A$ & 1.44 & 1.68 & 0.84 & 1.34 & -0.60 & -0.41 & \\
\hline$N=43$ & $(1.00)$ & $(0.00-8.00)$ & $(0.00)$ & $(0.00-7.00)$ & {$[-1.06,-0.15]$} & {$[-0.72 ;-0.10]$} & \\
\hline B & 1.63 & 1.41 & 1.00 & 1.10 & -0.63 & -0.49 & \\
\hline$N=24$ & $(1.00)$ & $(0.00-5.00)$ & $(1.00)$ & $(0.00-3.00)$ & {$[-1.22 ;-0.03]$} & {$[-0.86 ;-0.02]$} & \\
\hline C & 2.53 & 2.53 & 1.60 & 2.23 & -0.93 & -0.39 & \\
\hline$N=15$ & $(2.00)$ & $(0.00-7.00)$ & $(0.50)$ & $(0.00-7.00)$ & {$[-2.21 ;-0.35]$} & {$[-0.92 ; 0.13]$} & \\
\hline \multicolumn{8}{|c|}{ NRS “How intense is your dyspnea on exertion?” ( $0-10 \wedge)$} \\
\hline All patients & 5.56 & 2.50 & 3.41 & 2.73 & -2.15 & -0.82 & \multirow{8}{*}{$\begin{array}{l}F_{T}=39.0(1) \\
p<0.001 \\
F_{G}=0.25(2) \\
p=0.774 \\
F_{G^{\star} T}=65(2) \\
p=0.524\end{array}$} \\
\hline$N=82$ & $(6.00)$ & $(0.00-10.00)$ & $(3.00)$ & $(0.00-10.00)$ & {$[-2.73 ;-1.57]$} & {$[-1.01 ;-0.56]$} & \\
\hline A & 5.60 & 2.56 & 3.23 & 2.58 & -2.37 & -0.92 & \\
\hline$N=43$ & $(5.50)$ & $(0.00-10.00)$ & $(2.00)$ & $(0.00-10.00)$ & {$[-3.16 ;-1.58]$} & {$[-1.28 ;-0.56]$} & \\
\hline B & 5.46 & 2.47 & 3.29 & 2.66 & -2.17 & -0.85 & \\
\hline$N=24$ & $(6.00)$ & $(1.00-9.00)$ & $(2.00)$ & $(0.00-8.00)$ & {$[-3.37 ;-0.96]$} & {$[-1.21 ;-0.30]$} & \\
\hline C & 5.60 & 2.53 & 4.13 & 3.29 & -1.47 & -0.58 & \\
\hline$N=15$ & $(6.00)$ & $(0.00-9.00)$ & $(4.00)$ & $(0.00-10.00)$ & {$[-2.87 ;-0.06]$} & {$[-1.12 ;-0.02]$} & \\
\hline \multicolumn{8}{|c|}{$\operatorname{mMRC}(\vartheta 0-4 \wedge)$} \\
\hline All patients & 2.26 & 1.19 & 1.51 & 1.12 & -0.74 & -0.64 & \multirow{8}{*}{$\begin{array}{l}\mathrm{F}_{\mathrm{T}}=25.1(1), \\
\mathrm{p}<0.001 \\
\mathrm{~F}_{\mathrm{G}}=1.81(2), \\
\mathrm{p}=0.168 \\
\mathrm{~F}_{\mathrm{G}^{*} \mathrm{~T}}=1.14(2), \\
\mathrm{p}=0.323\end{array}$} \\
\hline$N=90$ & $(2.00)$ & $(0.00-4.00)$ & $(2.00)$ & $(0.00-4.00)$ & {$[-0.99 ;-0.50]$} & {$[-0.87 ;-0.42]$} & \\
\hline A & 2.52 & 1.29 & 1.60 & 1.09 & -0.92 & -0.76 & \\
\hline$N=48$ & $(2.00)$ & $(0.00-4.00)$ & $(2.00)$ & $(0.00-4.00)$ & {$[-1.27 ;-0.57]$} & {$[-1.08 ;-0.44]$} & \\
\hline B & 1.88 & 0.82 & 1.35 & 1.23 & -0.53 & -0.43 & \\
\hline$N=26$ & $(2.00)$ & $(0.00-4.00)$ & $(2.00)$ & $(0.00-4.00)$ & {$[-1.04 ;-0.04]$} & {$[-0.83 ;-0.03]$} & \\
\hline C & 2.06 & 1.24 & 1.50 & 1.10 & -0.56 & -0.69 & \\
\hline$N=16$ & $(2.00)$ & $(0.00-4.00)$ & $(1.50)$ & $(0.00-4.00)$ & {$[-1.00 ;-0.13]$} & {$[-1.23 ;-0.12]$} & \\
\hline
\end{tabular}

Exertional dyspnea was significantly more pronounced than resting dyspnea at baseline in all groups. In the overall group, moderate to large pre-post changes were observed for intensity in exertional dyspnea. A clinically relevant improvement of at least 2 points (MCID) was found in $66.1 \%$ of patients. $71.5 \%$ of the $50 \%$ of patients who reported NRS scores of $>6$ (median) at $T_{1}$ exhibited clinically relevant improvements at $\mathrm{T}_{2}$. There were small to moderate correlations between the improvement in exertional dyspnea and improvements in QoL, fatigue, and anxiety (see Table 3). 
Table 3

Correlations between the improvements in various parameters

\begin{tabular}{|c|c|c|c|c|c|c|c|c|c|c|c|c|c|c|c|}
\hline & NRS1 & NRS2 & mMRC & 6MWD & Vc & TLC & FEV1 & $\mathrm{PaO} 2$ & TLCO & PImax & $\begin{array}{l}\text { D- } \\
\text { dimers }\end{array}$ & CRP & $\begin{array}{l}\text { EQ-5D- } \\
5 \mathrm{~L}\end{array}$ & VAS & B \\
\hline NRS1 & 1 & $0.341^{* k}$ & 0.159 & $-0.223^{*}$ & 0.010 & 0.058 & 0.143 & -0.004 & 0.141 & 0.153 & -0.014 & -0.058 & 0.134 & 0.077 & 0 \\
\hline NRS2 & & 1 & 0.220 & 0.187 & 0.159 & 0.187 & 0.192 & -0.120 & 0.075 & 0.073 & -0.077 & -0.164 & $0.363^{* *}$ & $0.273^{*}$ & 0 \\
\hline mMRC & & & 1 & 0.074 & 0.099 & -0.127 & 0.092 & -0.038 & -0.012 & 0.030 & -0.058 & -0.050 & 0.164 & $0.405^{\star \star}$ & 0 \\
\hline 6MWD & & & & 1 & 0.065 & 0.058 & 0.027 & $0.281^{\star *}$ & 0.191 & $-0.280^{\star *}$ & 0.195 & $0.273^{\star}$ & $0.279^{*}$ & $0.244^{*}$ & 0 \\
\hline VC & & & & & 1 & $0.480^{\star \star}$ & $0.842^{\star \star}$ & $0.249^{*}$ & 0.126 & $0.338^{* *}$ & $0.295^{*}$ & 0.146 & 0.127 & 0.118 & 0 \\
\hline TLC & & & & & & 1 & $0.305^{* *}$ & $0.205^{*}$ & $0.227^{\star}$ & $0.222^{*}$ & $0.305^{*}$ & 0.108 & 0.199 & -0.020 & 0 \\
\hline FEV1 & & & & & & & 1 & $0.286^{\star \star}$ & $0.222^{*}$ & $0.248^{*}$ & 0.229 & 0.197 & 0.124 & 0.145 & 0 \\
\hline $\mathrm{PaO} 2$ & & & & & & & & 1 & $0.316^{\star *}$ & -0.068 & 0.165 & 0.281 & 0.006 & -0.128 & $-c$ \\
\hline TLCO & & & & & & & & & 1 & -0.003 & $0.306^{*}$ & 0.200 & 0.047 & 0.010 & 0 \\
\hline PImax & & & & & & & & & & 1 & 0.173 & 0.097 & -0.021 & 0.157 & $-c$ \\
\hline $\begin{array}{l}\text { D- } \\
\text { dimers }\end{array}$ & & & & & & & & & & & 1 & $0.600 * *$ & 0.162 & 0.155 & 0 \\
\hline CRP & & & & & & & & & & & & 1 & -0.092 & 0.102 & $-c$ \\
\hline $\begin{array}{l}\text { EQ-5D- } \\
5 \mathrm{~L}\end{array}$ & & & & & & & & & & & & & 1 & $0.296^{\star *}$ & 0 \\
\hline VAS & & & & & & & & & & & & & & 1 & 0 \\
\hline BFI & & & & & & & & & & & & & & & 1 \\
\hline
\end{tabular}

PHQ-9

GAD-7

Notes: Values in grey indicate nonsignificant results; values in bold indicate correlation coefficients of $\geq 0.3 ; *: p<0.05 ; * *$ : $p<0.01 ; 6 \mathrm{MWD}$ : 6-minute walking Inventory; CRP: C-reactive protein; EQ-5D: 5-level EQ-5D questionnaire; FEV1: Forced expiratory volume in one second: GAD-7: Generalized Anxiety Disorder-7; Research Council; NRS1: Numeric Rating Scale for resting dyspnea; NRS2: Numeric Rating Scale for exertional dyspnea; Pa02: partial pressure of 02; PHQ-9:

Questionnaire 9; PImax: maximal inspiratory pressure; TLC: total lung capacity; TLCO: diffusion capacity of the lungs for carbon monoxide; VAS: visual analo

For the mMRC scores, we also found moderate pre-post changes. Slightly more than $50 \%$ benefited from a clinically relevant improvement (MCID $=1$ level) without differences between the three subgroups. In total, $59 \%$ of the $50 \%$ of patients who were most severely affected (mMRC $>2$ ) benefited. There were small to moderate correlations between an improvement in mMRC scores and improvements in QoL (as measured by the VAS), fatigue, and anxiety (see Table 3).

Small to moderate changes were observed in the intensity of dyspnea at rest, with small correlations between an improvement in resting dyspnea and improvements in 6MWD and anxiety. We found no significant differences between the 3 subgroups in this regard, either at the start of PR or in the pre-post difference after PR. Moderate changes were also found for unpleasantness of dyspnea at rest or on exertion, which was slightly more pronounced at rest in group C (see Table S1 of the supplemental material).

\section{Secondary outcomes: objective parameters}

\section{Physical capacity}


Table 4

Results of physical capacity, pulmonary function, and laboratory blood tests

\begin{tabular}{|c|c|c|c|c|c|c|c|}
\hline Group & $\mathrm{T}_{1}$ & & $\mathrm{~T}_{2}$ & & & & \\
\hline & $\mathrm{M}$ & SD & $\mathrm{M}$ & SD & Delta & d & RM-ANOVA \\
\hline & (Median) & (Range) & (Median) & (Range) & {$[95 \% \mathrm{Cl}]$} & {$[95 \% \mathrm{Cl}]$} & $F(d f), p$ \\
\hline \multicolumn{8}{|c|}{ Physical capacity } \\
\hline \multicolumn{8}{|l|}{ 6-MWD [m] } \\
\hline All patients & 419 & 127 & 530 & 100 & 111 & 1.36 & \multirow{8}{*}{$\begin{array}{l}\mathrm{F}_{\mathrm{T}}=130.3(1) \\
\mathrm{p}=0.001 \\
\mathrm{~F}_{\mathrm{G}}=5.4(2) \\
\mathrm{p}=0.006 \\
\mathrm{~F}_{\mathrm{G}^{*} \mathrm{~T}}=4.1(2) \\
\mathrm{p}=0.019\end{array}$} \\
\hline$N=97$ & $(450)$ & $(13-663)$ & $(530)$ & $(175-740)$ & {$[94 ; 127]$} & {$[1.08 ; 1.63]$} & \\
\hline A & 377 & 142 & 508 & 111 & 131 & 1.52 & \\
\hline$N=52$ & $(392)$ & $(13-603)$ & (522) & $(175-720)$ & {$[107 ; 155]$} & {$[1.12 ; 1.92]$} & \\
\hline B & 459 & 79 & 555 & 79.5 & 96 & 1.61 & \\
\hline$N=27$ & $(470)$ & $(270-590)$ & $(564)$ & $(406-675)$ & {$[72 ; 120]$} & {$[1.02 ; 2.17]$} & \\
\hline C & 480 & 96.8 & 554 & 85.9 & 74 & 0.91 & \\
\hline$N=18$ & $(479)$ & $(275-663)$ & $(544)$ & $(380-740)$ & {$[33 ; 114]$} & {$[0.35 ; 1.45]$} & \\
\hline \multicolumn{8}{|c|}{ Lung function } \\
\hline \multicolumn{8}{|c|}{ Vital capacity (VC) [ \%pred.] } \\
\hline All patients & 89.6 & 20.7 & 98.0 & 16.2 & 8.3 & 0.47 & \multirow{8}{*}{$\begin{array}{l}F_{T}=9.49(1) \\
p=0.003 \\
F_{G}=12.0(2) \\
p<0.001 \\
F_{G^{\star} T}=4.77(2) \\
p=0.011\end{array}$} \\
\hline$N=103$ & $(90.9)$ & $(33.7-130.1)$ & $(100.3)$ & $(63.4-129.3)$ & {$[4.9 ; 11.8]$} & {$[0.27 ; 0.67]$} & \\
\hline A & 81.1 & 21.7 & 93.6 & 16.5 & 12.5 & 0.66 & \\
\hline$N=54$ & $(79.7)$ & $(33.7-130.1)$ & $(96.6)$ & $(63.4-129.3)$ & {$[7.3 ; 17.7]$} & {$[0.36 ; 0.95]$} & \\
\hline B & 95.3 & 14.4 & 102.0 & 14.5 & 6.7 & 0.39 & \\
\hline$N=32$ & $(97.5)$ & $(63.0-115.5)$ & $(103.6)$ & $(64.1-122.7)$ & {$[0.5 ; 12.8]$} & {$[0.03 ; 0.75]$} & \\
\hline C & 106.3 & 12.9 & 104.5 & 14.9 & -1.8 & -0.21 & \\
\hline$N=17$ & $(104.2)$ & $(85.4-129.6)$ & $(106.0)$ & $(68.4-125.5)$ & {$[-6.27 ; 2.64]$} & {$[-0.69 ; 0.27]$} & \\
\hline \multicolumn{8}{|c|}{ Total lung capacity (TLC) [\%pred.] } \\
\hline All patients & 92.8 & 17.3 & 97.7 & 14.9 & 4.9 & 0.36 & \multirow{8}{*}{$\begin{array}{l}F_{T}=5.61(1), \\
p=0.020 \\
F_{G}=14.8(2) \\
p<0.001 \\
F_{G^{\star} T}=2.47(2), \\
p=0.089\end{array}$} \\
\hline$N=103$ & $(95.6)$ & $(46.8-134.1)$ & $(99.1)$ & $(63.7-128.1)$ & {$[2.3 ; 7.6]$} & {$[0.16 ; 0.56]$} & \\
\hline A & 85.4 & 17.0 & 92.0 & 13.8 & 6.5 & 0.47 & \\
\hline$N=54$ & $(86.3)$ & $(46.8-121.4)$ & $(91.1)$ & $(63.7-119.6)$ & {$[2.7 ; 10.4]$} & {$[0.18 ; 0.74]$} & \\
\hline B & 98.3 & 13.9 & 104.1 & 14.8 & 5.8 & 0.41 & \\
\hline$N=32$ & $(98.1)$ & $(70.8-125.7)$ & $(106.0)$ & $(72.4-128.1)$ & {$[0.6 ; 10.9]$} & {$[0.04 ; 0.76]$} & \\
\hline$C$ & 105.5 & 12.0 & 103.8 & 11.1 & -1.7 & -0.17 & \\
\hline$N=17$ & $(104.1)$ & $(81.1-134.1)$ & (101.6) & $(81.6-126.9)$ & {$[-6.9 ; 3.4]$} & {$[-0.65 ; 0.31]$} & \\
\hline \multicolumn{8}{|c|}{ Forced expiratory volume in in one second (FEV1) [\%pred.] } \\
\hline All patients & 92.3 & 20.8 & 100.9 & 16.5 & 8.6 & 0.49 & \multirow{6}{*}{$\begin{array}{l}F_{T}=12.6(1) \\
p<0.001 \\
F_{G}=7.9(2) \\
p<0.001 \\
F_{G^{*} T}=2.88(2), \\
p=0.061\end{array}$} \\
\hline$N=103$ & $(92.0)$ & $(23.2-135.7)$ & (103.3) & $(36.7-138.1)$ & {$[5.2 ; 12.0]$} & {$[0.29 ; 0.70]$} & \\
\hline A & 85.0 & 23.1 & 97.2 & 18.2 & 12.16 & 0.59 & \\
\hline$N=54$ & (85.3) & $(23.2-135.7)$ & $(97.0)$ & $(36.7-138.1)$ & {$[6.5 ; 17.8]$} & {$[0.29 ; 0.87]$} & \\
\hline B & 97.3 & 14.0 & 103.7 & 13.4 & 6.4 & 0.46 & \\
\hline$N=32$ & $(96.8)$ & $(69.1-126.2)$ & (106.4) & $(72.5-131.3)$ & {$[1.4 ; 11.5]$} & {$[0.09 ; 0.82]$} & \\
\hline
\end{tabular}




\begin{tabular}{|c|c|c|c|c|c|c|c|}
\hline Group & $\mathrm{T}_{1}$ & & $\mathrm{~T}_{2}$ & & & & \\
\hline C & 105.8 & 13.4 & 107.3 & 13.4 & 1.5 & 0.25 & \\
\hline$N=17$ & $(106.0)$ & $(86.7-129.7)$ & $(108.7)$ & $(76.6-131.2)$ & {$[-1.6 ; 4.5]$} & {$[-0.23 ; 0.72]$} & \\
\hline \multicolumn{8}{|c|}{ Partial pressure of $02(\mathrm{PaO} 2)[\mathrm{mm} \mathrm{Hg}]$} \\
\hline All patients & 74.4 & 9.0 & 78.4 & 7.1 & 4.0 & 0.55 & \multirow{8}{*}{$\begin{array}{l}F_{T}=1571.9(1), \\
p<0.001 \\
F_{G}=4.7(2), \\
p=0.02 \\
F_{G^{\star} T}=6.1(2), \\
p=0.003\end{array}$} \\
\hline$N=100$ & $(74.5)$ & $(43.0-98.0)$ & $(78.0)$ & $(58.0-95.0)$ & {$[2.6 ; 5.5]$} & {$[0.34 ; 0.76]$} & \\
\hline A & 71.7 & 9.6 & 77.3 & 7.4 & 5.6 & -0.63 & \\
\hline$N=51$ & $(72.0)$ & $(43.0-88.0)$ & $(78.0)$ & $(58.0-94.0)$ & {$[3.7 ; 7.5]$} & {$[0.50 ; 1.14]$} & \\
\hline B & 75.6 & 7.2 & 79.4 & 6.6 & 3.7 & 0.49 & \\
\hline$N=31$ & $(73.0)$ & $(66.0-92.0)$ & $(78.0)$ & $(70.0-95.0)$ & {$[0.9 ; 6.5]$} & {$[0.12 ; 0.86]$} & \\
\hline C & 79.9 & 7.3 & 80.0 & 6.7 & 0.06 & 0.01 & \\
\hline$N=18$ & $(79.5)$ & $(69.0-98.0)$ & $(79.5)$ & $(71.0-91.0)$ & {$[3.4 ;-3.5]$} & {$[-0.45 ; 0.47]$} & \\
\hline \multicolumn{8}{|c|}{ Diffusion capacity of the lungs for carbon monoxide (TLCO SB) [\%pred.] } \\
\hline All patients & 73.8 & 22.0 & 80.0 & 20.3 & 6.2 & 0.58 & \multirow{8}{*}{$\begin{array}{l}\mathrm{F}_{\mathrm{T}}=13.4(1) \\
\mathrm{p}<0.001 \\
\mathrm{~F}_{\mathrm{G}}=11.1(2) \\
\mathrm{p}<0.001 \\
\mathrm{~F}_{\mathrm{G}^{\star} \mathrm{T}}=8.0(2) \\
\mathrm{p}<0.001\end{array}$} \\
\hline$N=93$ & $(77.1)$ & $(11.7-124.0)$ & $(78.4)$ & $(35.1-120.2$ & {$[4.0 ; 8.4]$} & {$[0.36 ; 0.79]$} & \\
\hline A & 63.6 & 21.1 & 73.4 & 19.7 & 9.9 & 0.91 & \\
\hline$N=48$ & $(61.1)$ & $(11.7-105.2)$ & $(73.9)$ & $(38.0-114.8)$ & {$[6.7 ; 13.1]$} & {$[0.57 ; 1.24]$} & \\
\hline$B$ & 81.4 & 18.3 & 85.2 & 20.6 & 3.8 & -0.43 & \\
\hline$N=30$ & $(84.6)$ & $(32.7-107.6)$ & $(82.9)$ & $(35.1-120.2)$ & {$[0.48 ; 7.1]$} & {$[0.05 ; 0.80]$} & \\
\hline $\mathrm{C}$ & 91.6 & 12.9 & 90.6 & 14.0 & -1.0 & -0.11 & \\
\hline$N=15$ & $(90.7)$ & $(70.2-124.0)$ & $(92.4)$ & $(65.9-118.2)$ & {$[-5.9 ; 3.9]$} & {$[-0.62 ; 0.40]$} & \\
\hline \multicolumn{8}{|c|}{ Maximal inspiratory pressure (PImax) [\%pred.] } \\
\hline All patients & 62.4 & 25.9 & 70.1 & 24.7 & 7.7 & 0.36 & \multirow{8}{*}{$\begin{array}{l}\mathrm{F}_{\mathrm{T}}=8.85(1), \\
\mathrm{p}=0.004 \\
\mathrm{~F}_{\mathrm{G}}=2.6(2), \\
\mathrm{p}=0.083 \\
\mathrm{~F}_{\mathrm{G}^{*} \mathrm{~T}}=0.98(2), \\
\mathrm{p}=0.38\end{array}$} \\
\hline$N=98$ & $(59.7)$ & $(6.6-133.3)$ & $(67.4)$ & $(19.9-133.3)$ & {$[3.4 ; 12.0]$} & {$[0.15 ; 0.56]$} & \\
\hline A & 64.5 & 27.6 & 70.9 & 23.4 & 6.3 & 0.34 & \\
\hline$N=52$ & $(63.0)$ & $(6.6-133.3)$ & $(68.9)$ & $(19.9-131.0)$ & {$[1.1 ; 11.5]$} & {$[0.06 ; 0.61]$} & \\
\hline B & 63.5 & 26.5 & 75.5 & 28.6 & 12.0 & 0.43 & \\
\hline$N=31$ & $(61.9)$ & $(20.1-116.3)$ & $(69.6)$ & $(21.5-133.3)$ & {$[1.7 ; 22.3]$} & {$[0.05 ; 0.79]$} & \\
\hline C & 52.4 & 15.3 & 56.1 & 14.7 & 3.7 & 0.30 & \\
\hline$N=15$ & $(54.0)$ & $(27.7-88.2)$ & $(53.3)$ & $(25.8-88.2)$ & {$[-3.2 ; 10.6]$} & {$[-0.23 ; 0.81]$} & \\
\hline \multicolumn{8}{|c|}{ Laboratory blood tests } \\
\hline \multicolumn{8}{|c|}{ D-dimers [ng/ml] (Normal value < 500 ng/ml) } \\
\hline All patients & 1082.2 & 1329.5 & 614.0 & 578.3 & -468.2 & -0.43 & \multirow{8}{*}{$\begin{array}{l}\mathrm{F}_{\mathrm{T}}=3.48(1), \\
\mathrm{p}=0.07 \\
\mathrm{~F}_{\mathrm{G}}=3.93(2), \\
\mathrm{p}=0.024 \\
\mathrm{~F}_{\mathrm{G}^{* \top}}=5.32(2), \\
\mathrm{p}=0.007\end{array}$} \\
\hline$N=69$ & $(517.0)$ & $(173.0-6824.0)$ & $(414.0)$ & $(182.0-2931.0)$ & {$[-734.9 ;-201.5]$} & {$[-0.67 ;-0.17]$} & \\
\hline A & 1490.9 & 1571.4 & 725.8 & 611.6 & -765.1 & -0.56 & \\
\hline$N=41$ & $(866.0)$ & $(197.0-6824.0)$ & $(539.0)$ & $(182.0-2931.0)$ & {$[-1194.9 ;-335.2]$} & {$[-0.89 ;-0.23]$} & \\
\hline B & 536.9 & 502.6 & 516.7 & 601.5 & -20.3 & -0.11 & \\
\hline$N=18$ & $(383.0)$ & $(173.0-2308.0)$ & $(358.0)$ & $(213.0-2826.0)$ & {$[-111.5 ; 71.0]$} & {$[-0.35 ; 0.57]$} & \\
\hline C & 387.9 & 108.0 & 330.8 & 127.3 & -57.1 & -0.46 & \\
\hline$N=10$ & (385.5) & $(265.0-583.0)$ & $(283.5)$ & $(226.0-571.0)$ & {$[-146.6 ; 32.4]$} & {$[-1.10 ; 0.21]$} & \\
\hline
\end{tabular}

Notes: 6-MWD: 6 Minute Walking Distance Test; $T_{1}$ : start of rehabilitation; $T_{2}$ : end of rehabilitation; M: mean; SD: standard deviation; Delta: difference $\mathrm{M}_{\mathrm{T} 2}$

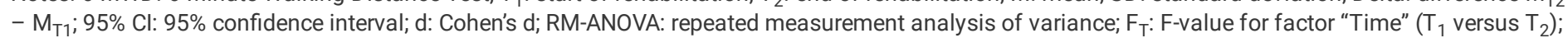
FG: $F$ value for factor "Group" (A versus B versus C); FG*T: F value for interaction of "Time” and "Group” 


\begin{tabular}{|c|c|c|c|c|c|c|c|}
\hline Group & $\mathrm{T}_{1}$ & & $\mathrm{~T}_{2}$ & & & & \\
\hline All patients & 5.86 & 5.62 & 4.10 & 4.08 & -1.77 & -0.41 & $\mathrm{~F}_{\mathrm{T}}=4.8(1)$ \\
\hline$N=71$ & (3.70) & $(0.50-27.70)$ & $(2.80)$ & $(0.60-20.80)$ & {$[-2.78 ; 0.75]$} & {$[-0.65 ;-0.16]$} & \multirow{7}{*}{$\begin{array}{l}\mathrm{p}=0.032 \\
\mathrm{~F}_{\mathrm{G}}=1.4(2), \\
\mathrm{p}=0.251 \\
\mathrm{~F}_{\mathrm{G}^{\star} \mathrm{T}}=1.8(2), \\
\mathrm{p}=0.178\end{array}$} \\
\hline A & 7.01 & 6.60 & 4.47 & 4.69 & -2.54 & 0.50 & \\
\hline$N=39$ & $(4.20)$ & $(0.80-27.70)$ & (2.70) & $(0.60-20.80)$ & {$[-4.19 ; 0.90]$} & {$[-0.83 ;-0.17]$} & \\
\hline B & 4.93 & 4.15 & 3.69 & 3.08 & -1.25 & -0.37 & \\
\hline$N=21$ & (3.50) & $(0.90-13.70)$ & (3.10) & $(0.60-11.00)$ & {$[-2.78 ; 0.29]$} & {$[-0.81 ; 0.08]$} & \\
\hline C & 3.55 & 2.89 & 3.56 & 3.57 & 0.02 & 0.01 & \\
\hline$N=11$ & (3.10) & $(0.50-8.90)$ & $(3.00)$ & $(0.60-12.50)$ & {$[-1.15 ; 1.18]$} & {$[-0.58 ; 0.60]$} & \\
\hline
\end{tabular}

The 6MWD improved significantly with large effect sizes over the course of PR in all groups, with patients in subgroups A and B showing even larger changes than those in group $\mathrm{C}$. There were also significant differences concerning the improvements between the subgroups, indicating that patients who were more severely burdened at $\mathrm{T}_{1}$ achieved a higher improvement. Overall, significant reductions in physical performance compared to a healthy reference population matched by age, sex, and BMI [31] were observed. Group A showed the largest deviation of -59.22 \%pred. Group B showed a percentage deviation of -31.41 \%pred., and group C showed a percentage deviation of $-16.45 \%$ pred.

\section{Lung function}

A total of $34.6 \%$ of the overall patients had a restrictive lung function pattern (inspiratory vital capacity (IVC) $<80 \%$ ) at $\mathrm{T}_{1}$ (group A $54.6 \%$, group B $21.9 \%$, group C $0 \%$ ), but only $17.3 \%$ of patients had a restrictive lung function pattern at $\mathrm{T}_{2}$ (group A $12.6 \%$, group B $12.5 \%$ ). In terms of VC\%pred., the total group improved by $8.3 \%$ (group A $12.5 \%$, group B: $6.7 \%$ ). Lung diffusion, which was slightly impaired at baseline, improved in the overall group, with a moderate effect size. $A$ total of $56.3 \%$ of all patients had a pathological lung transfer factor for $\mathrm{CO}$ (TLCO single breath $<80 \%$ pred.) at $T_{1}(A=78.9 \%, B=43.8 \%, C=$ 15.0\%). At $\mathrm{T}_{2}$, the prevalence decreased to $52.1 \%$ (group A showed a reduction to only $62.8 \%$ ). At $\mathrm{T}_{1}$, group $A$ exhibited significantly greater impairment in several tests of lung function, blood gas and lung diffusion, such as VC, RV, TLC, FEV1, PaO2, and TLCO. Groups A and B showed statistically significant improvements with moderate to high effect sizes in VC\%, TLC\%, FEV1\%, TLCO_SB\%, and PImax, while group C, which already showed normal values in this respect at $T_{1}$, showed no improvement in these parameters.

\section{Laboratory test results}

D-dimers of group A were on average significantly elevated. D-dimers and CRP declined during PR in groups A and B. No changes were observed in BNP or serum LDH levels (see Table S2 of the supplemental material).

\section{Secondary outcomes: Patient-reported outcomes}

\section{NRS cough, sputum, and pain}

Patients reported only low levels of cough and phlegm at $\mathrm{T}_{1}$, with small to moderate changes in all groups (see Table $\mathrm{S} 1$ of the supplemental material). The experience of pain was most pronounced in group $\mathrm{C}$, with small reductions in the overall group. 
Table 5

Results of patient-reported outcomes

\begin{tabular}{|c|c|c|c|c|c|c|c|}
\hline Group & $\mathrm{T}_{1}$ & & $\mathrm{~T}_{2}$ & & & & \\
\hline & 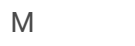 & SD & M & SD & Delta & d & RM-ANOVA \\
\hline & (Median) & (Range) & (Median) & (Range) & {$[95 \% \mathrm{Cl}]$} & {$[95 \% \mathrm{Cl}]$} & $F(d f), p$ \\
\hline \multicolumn{8}{|c|}{ Quality of life } \\
\hline \multicolumn{8}{|c|}{ EQ-5D-5L ( } \\
\hline All patients & 11.65 & 3.16 & 9.23 & 3.02 & -2.41 & -0.95 & \multirow{8}{*}{$\begin{array}{l}F_{T}=52.6(1) \\
p<0.001 \\
F_{G}=0.803(2) \\
p=0.452 \\
F_{G^{*} T}=1.81(2) \\
p=0.169\end{array}$} \\
\hline$N=82$ & $(12.00)$ & $(5.00-20.00)$ & $(8.50)$ & $(5.00-16.00)$ & {$[-2.98 ;-1.85]$} & {$[-1.21 ;-0.68]$} & \\
\hline A & 11.60 & 2.93 & 8.67 & 2.82 & -2.93 & -1.17 & \\
\hline$N=42$ & $(12.00)$ & $(5.00-18.00)$ & $(8.00)$ & $(5.00-15.00)$ & {$[-3.71 ;-2.15]$} & {$[-1.56 ;-0.77]$} & \\
\hline B & 11.48 & 3.04 & 9.52 & 2.84 & -1.96 & -0.90 & \\
\hline$N=25$ & $(12.00)$ & $(5.00-17.00)$ & $(9.00)$ & $(5.00-15.00)$ & {$[-2.86 ;-1.06]$} & {$[-1.36 ;-0.42]$} & \\
\hline C & 12.07 & 4.06 & 10.33 & 3.62 & -1.73 & -0.56 & \\
\hline$N=15$ & $(11.50)$ & $(6.00-20.00)$ & $(11.00)$ & $(5.00-16.00)$ & {$[-3.44 ;-0.03]$} & {$[-1.10 ;-0.01]$} & \\
\hline \multicolumn{8}{|c|}{ EQ-5D-5L-VAS (૭ 100-0 ^) } \\
\hline All patients & 50.01 & 17.03 & 68.05 & 16.07 & 18.04 & 1.09 & \multirow{8}{*}{$\begin{array}{l}F_{T}=86.4(1) \\
p<0.001 \\
F_{G}=5.64(2) \\
p=0.005 \\
F_{G^{\star} T}=1.23(2) \\
p=0.296\end{array}$} \\
\hline$N=83$ & $(50.00)$ & $(0.00-90.00)$ & $(70.00)$ & $(25.00-95.00)$ & {$[14.6 ; 21.4]$} & {$[0.88 ; 1.44]$} & \\
\hline A & 49.53 & 17.28 & 70.12 & 15.59 & 20.58 & 1.30 & \\
\hline$N=43$ & $(50.00)$ & $(0.00-90.00)$ & $(70.00)$ & $(30.00-95.00)$ & {$[15.7 ; 25.5]$} & {$[0.89 ; 1.70]$} & \\
\hline$B$ & 56.71 & 12.07 & 71.50 & 12.15 & 14.79 & 0.99 & \\
\hline$N=24$ & $(57.50)$ & $(24.00-80.00)$ & $(72.50)$ & $(50.00-90.00)$ & [8.45; 21.13] & {$[0.49 ; 1.47]$} & \\
\hline C & 41.25 & 19.28 & 57.31 & 18.75 & 16.06 & 1.05 & \\
\hline$N=16$ & $(35.00)$ & $(15.00-80.00)$ & $(60.00)$ & $(25.00-85.00)$ & {$[7.92 ; 24.20]$} & {$[0.43 ; 1.66]$} & \\
\hline \multicolumn{8}{|l|}{ Fatigue } \\
\hline \multicolumn{8}{|c|}{$\mathrm{BFI}(\vartheta 0-10 \wedge)$} \\
\hline All patients & 4.39 & 2.05 & 2.69 & 2.08 & -1.70 & -0.99 & \multirow{8}{*}{$\begin{array}{l}F_{T}=54.4(1) \\
p<0.001 \\
F_{G}=5.76(2) \\
p=0.005 \\
F_{G^{*} T}=3.41(2) \\
p=0.041\end{array}$} \\
\hline$N=81$ & $(4.33)$ & $(0.00-8.56)$ & $(2.11)$ & $(0.00-8.33)$ & {$[-2.08 ;-1.32]$} & {$[-1.25 ;-0.72]$} & \\
\hline$A$ & 4.05 & 1.88 & 1.92 & 1.43 & -2.13 & -1.24 & \\
\hline$N=82$ & $(4.13)$ & $(0.00-8.00)$ & $(1.40)$ & $(0.00-5.56)$ & {$[-2.59 ;-1.66]$} & {$[-1.87 ;-0.10]$} & \\
\hline B & 4.47 & 2.19 & 3.03 & 2.16 & -1.44 & -0.85 & \\
\hline$N=24$ & $(4.78)$ & $(0.22-8.56)$ & $(2.61)$ & $(0.11-7.56)$ & {$[-2.16 ;-0.72]$} & {$[-1.31 ;-0.37]$} & \\
\hline C & 5.21 & 2.15 & 4.30 & 2.50 & -0.91 & -0.43 & \\
\hline$N=15$ & $(4.56)$ & $(2.11-8.44)$ & (3.78) & $(0.67-8.33)$ & {$[-2.07 ; 0.25]$} & {$[-0.96 ; 0.10]$} & \\
\hline \multicolumn{8}{|c|}{ Depression and anxiety } \\
\hline \multicolumn{8}{|c|}{ PHQ-9 (૭ 0-27 ^) } \\
\hline All patients & 9.95 & 5.28 & 5.68 & 4.20 & -4.27 & -1.18 & \multirow{6}{*}{$\begin{array}{l}F_{T}=75.2(1) \\
p<0.001 \\
F_{G}=1.26(2) \\
p=0.289 \\
F_{G^{*} T}=1.22(2) \\
p=0.299\end{array}$} \\
\hline$N=78$ & $(10.00)$ & $(0.00-22.00)$ & $(4.00)$ & $(0.00-18.00)$ & {$[-5.09 ;-3.45]$} & {$[-1.47 ;-0.89]$} & \\
\hline A & 9.67 & 5.44 & 4.83 & 3.58 & -4.83 & -1.34 & \\
\hline$N=42$ & $(9.00)$ & $(0.00-22.00)$ & $(4.00)$ & $(0.00-14.00)$ & {$[-5.96 ;-3.71]$} & {$[-1.75 ;-0.92]$} & \\
\hline B & 9.83 & 5.11 & 6.00 & 4.34 & -3.83 & -1.06 & \\
\hline$N=23$ & $(10.00)$ & $(1.00-22.00)$ & $(5.00)$ & $(0.00-16.00)$ & {$[-5.39 ;-2.26]$} & {$[-1.56 ;-0.54]$} & \\
\hline \multicolumn{8}{|c|}{$\begin{array}{l}\text { Notes: BFI: Brief Fatigue Inventory; EQ-5D-5L: 5-level EQ-5D questionnaire; GAD-7: Generalized Anxiety Disorder-7; PHQ-9: Patient Health Questionnaire 9; } \\
\text { VAS: visual analog scale; NRS: Numeric Rating Scale; } \mathrm{T}_{1} \text { : start of rehabilitation; } \mathrm{T}_{2} \text { : end of rehabilitation; M: mean; SD: standard deviation; Delta: difference } \\
\left.\mathrm{M}_{\mathrm{T} 2}-\mathrm{M}_{\mathrm{T} 1} ; 95 \% \text { Cl: } 95 \% \text { confidence interval; d: Cohen's d; RM-ANOVA: repeated-measures analysis of variance; } \mathrm{F}_{\mathrm{T}}: \mathrm{F}-\mathrm{value} \text { for factor "Time" ( } \mathrm{T}_{1} \text { versus } \mathrm{T}_{2}\right) \text {; } \\
\text { FG: } \mathrm{F} \text { value for factor "Group" (A versus B versus C); FG*T: F value for interaction of "Time" and "Group"; }\end{array}$} \\
\hline
\end{tabular}




\begin{tabular}{|c|c|c|c|c|c|c|c|}
\hline Group & $\mathrm{T}_{1}$ & & $\mathrm{~T}_{2}$ & & & & \\
\hline C & 11.08 & 5.31 & 7.85 & 5.21 & -3.23 & \multicolumn{2}{|l|}{-0.90} \\
\hline$N=13$ & $(11.00)$ & $(1.00-20.00)$ & $(6.00)$ & $(1.00-18.00)$ & {$[-5.40 ;-1.06\}$} & \multicolumn{2}{|l|}{$[0.24 ; 1.54]$} \\
\hline \multicolumn{8}{|c|}{ GAD-7 $(\vartheta 0-21 \wedge)$} \\
\hline All patients & 6.39 & 4.92 & 4.00 & 3.83 & -2.39 & -0.66 & \multirow{8}{*}{$\begin{array}{l}F_{T}=22.1(1), \\
p<0.001 \\
F_{G}=0.939(2), \\
p=0.396 \\
F_{G^{*} T}=1.62(2), \\
p=0.205\end{array}$} \\
\hline$N=77$ & $(5.00)$ & $(0.00-21.00)$ & $(3.00)$ & $(0.00-15.00)$ & {$[-3.22 ;-1.56]$} & {$[-0.90 ;-0.41]$} & \\
\hline A & 6.30 & 5.44 & 3.40 & 3.17 & -2.90 & -0.80 & \\
\hline$N=40$ & $(5.00)$ & $(0.00-21.00)$ & $(3.00)$ & $(0.00-12.00)$ & {$[-4.05 ;-1.75]$} & {$[-1.16 ;-0.44]$} & \\
\hline B & 6.18 & 3.80 & 3.73 & 3.97 & -2.45 & -0.79 & \\
\hline$N=22$ & $(6.00)$ & $(0.00-13.00)$ & $(3.00)$ & $(0.00-15.00)$ & [-1.07: -0.66] & {$[-1.26 ;-0.30]$} & \\
\hline C & 6.93 & 5.19 & 6.00 & 4.75 & -0.93 & -0.22 & \\
\hline$N=15$ & $(7.00)$ & $(0.00-16.00)$ & $(7.00)$ & $(0.00-15.00)$ & {$[-3.30 ; 1.43]$} & {$[-0.73 ; 0.30]$} & \\
\hline \multicolumn{8}{|c|}{$\begin{array}{l}\text { Notes: BFI: Brief Fatigue Inventory; EQ-5D-5L: 5-level EQ-5D questionnaire; GAD-7: Generalized Anxiety Disorder-7; PHQ-9: Patient Health Questionnaire 9; } \\
\text { VAS: visual analog scale; NRS: Numeric Rating Scale; } \mathrm{T}_{1} \text { : start of rehabilitation; } \mathrm{T}_{2} \text { : end of rehabilitation; M: mean; SD: standard deviation; Delta: difference } \\
\mathrm{M}_{\mathrm{T} 2}-\mathrm{M}_{\mathrm{T} 1} ; 95 \% \text { Cl: } 95 \% \text { confidence interval; } \mathrm{d} \text { : Cohen's d; RM-ANOVA: repeated-measures analysis of variance; } \mathrm{F}_{\mathrm{T}} \text { : F-value for factor "Time" ( } \mathrm{T}_{1} \text { versus } \mathrm{T}_{2} \text { ); } \\
\text { FG: F value for factor "Group" (A versus B versus C); FG*T: F value for interaction of "Time" and "Group"; }\end{array}$} \\
\hline
\end{tabular}

\section{Quality of life}

Patients in groups A and B showed higher QoL than patients in group C, as measured using the VAS. All groups showed significant improvement with high effect sizes, according to both an increase in the EQ-5D-VAS and a decrease in EQ-5D-5L. The MCID of the VAS between two measurements was 8 points. This was achieved at $\mathrm{T}_{2}$ in groups $\mathrm{A}, \mathrm{B}$, and $\mathrm{C}$ by $81.4 \%, 70.8 \%$, and $62.5 \%$ of patients, respectively. Even though the increase in VAS scores was not intercorrelated with the rating of the general efficacy of the PR $(r=0.182, p=0.109)$, we detected a significant correlation with the first GROC value $(r=0.360, p=0.001)$. In the overall group, the improvement in VAS scores correlated significantly with a decrease in mMRC, the intensity of exertional dyspnea, fatigue, and anxiety, and with an increase in the 6MWD.

\section{Fatigue}

A significant decrease in fatigue was observed in groups $A$ and $B$, with large effect sizes. Fatigue was more pronounced in group $C$ at $T_{1}$, and the decrease over the course of PR did not reach statistical significance. To illustrate the findings regarding the differences in fatigue in the 3 subgroups, the relative frequencies of fatigue severity are displayed in Fig. 3.

The overall decrease in fatigue was significantly intercorrelated with decreases in dyspnea, as measured by both the NRS and the mMRC. Furthermore, significant correlations were found for decreases in depression, anxiety, and impairments in QoL.

\section{Depression and anxiety}

At $T_{1}$, the mean values of the PHQ-9 were just below the cutoff between mild and moderate depressive symptom severity (10 points), with $52.6 \%$ showing a value of $\geq 10$ and $16.5 \%$ showing a value of $\geq 15$, indicating moderately severe depressive psychopathology. Mean values on the PHQ-9 declined with high effect sizes in all groups, and at $\mathrm{T}_{2}$, only $22.6 \%$ (2.4\%) showed values $\geq 10$ points ( $\geq 15$ points). Anxiety scores at $\mathrm{T}_{1}$ indicated, on average, mild psychopathology ( 5 to 9 points on the GAD-7). However, approximately one-third of the sample ( $29.3 \%$ ) showed scores of $\geq 10$. Anxiety values declined, with moderate to high effect sizes. At $\mathrm{T}_{2}$ only $8.7 \%$ showed values $\geq 10$ points. There were strong correlations between a decrease in fatigue and reductions in both depression and anxiety (see Table 3).

Rating of the overall effectiveness of PR from the patient's perspective and global rating of change in subjective health

Patients rated the effectiveness of PR as high, ranging from 7.07 in group C to 8.93 in group A (see Table 6). Significant differences could be detected between group A and group C, with group A reporting higher effectiveness of PR.

Table 6: Patients' rating of the rehabilitation efficacy. 


\begin{tabular}{|c|c|c|c|c|c|c|}
\hline \multirow[t]{3}{*}{ Group } & \multicolumn{2}{|c|}{$\begin{array}{l}\text { "How effective was the rehabilitation } \\
\text { program for you?" } \\
\text { L } 0-10 \mathrm{~J}\end{array}$} & \multicolumn{2}{|c|}{$\begin{array}{l}\text { "Compared to the time just before I started } \\
\text { rehabilitation, my health is now:" } \\
\text { very much worse }(-7)-\text { unchanged }(0) \text { - very much } \\
\text { better }(+7)\end{array}$} & \multicolumn{2}{|c|}{$\begin{array}{l}\text { "Compared to the time before my Corona } \\
\text { infection, my health is now:" } \\
\text { very much worse }(-7) \text { - unchanged }(0) \text { - very } \\
\text { much better }(+7)\end{array}$} \\
\hline & M & SD & M & SD & M & SD \\
\hline & (Median) & (Range) & (Median) & (Range) & (Median) & (Range) \\
\hline \multirow{2}{*}{$\begin{array}{l}\text { All } \\
\text { patients }\end{array}$} & 8.28 & 1.96 & 4.34 & 2.08 & -1.56 & 3.59 \\
\hline & $(9.00)$ & $0.00-10.00$ & $(5.00)$ & $-6.00-7.00$ & -3.00 & $-7.00-7.00$ \\
\hline \multirow[t]{2}{*}{ A } & 8.93 & 1.42 & 5.16 & 1.48 & -0.53 & 3.83 \\
\hline & (9.50) & $4.00-10.00$ & $(5.00)$ & $0.00-7.00$ & -1.00 & $-7.00-7.00$ \\
\hline \multirow[t]{2}{*}{ B } & 7.78 & 1.64 & 4.04 & 1.37 & -1.88 & 2.52 \\
\hline & (8.00) & $4.00-10.00$ & $(4.00)$ & $-1.00-7.00$ & -3.00 & $-5.00-5.00$ \\
\hline \multirow[t]{2}{*}{ C } & 7.07 & 2.87 & 2.81 & 2.21 & -4.25 & 3.01 \\
\hline & (8.00) & $0.00-10.00$ & (3.50) & $-6.00-6.00$ & -5.00 & $-7.00-4.00$ \\
\hline
\end{tabular}

Notes: M: mean; SD: standard deviation

Regarding GROC values, patients reported, on average, an improvement in health over the course of PR but still reported a decrease in health when comparing the status at $\mathrm{T}_{2}$ with the status before SARS-CoV-2 infection.

\section{GROC compared to the beginning of PR}

A strong improvement ( +6 to +7 points) was reported by $40.0 \%$ of group $A, 16.0 \%$ of group B, and $12.5 \%$ of group $C$, and a moderate improvement ( +4 to +5 points) was reported by $46.7 \%, 52.0 \%$ and $37.6 \%$ of groups $A, B$, and $C$, respectively. A weak but significant correlation was found between the GROC values for the duration of treatment and the reduction of dyspnea on exertion $(r=0.259, p=0.019)$, the changes in mMRC values $(r=0.252, p=0.029)$, and the increase in the 6MWD $(r=0.323, p=0.003)$. A stronger correlation was found between the GROC values for the duration of treatment and the reduction in fatigue ( $r=$ $0.429, p<0.001)$.

\section{Safety and feasibility of PR in patients after COVID-19}

During PR, complications were recorded in a systematic and standardized way. Of the 108 patients, one patient had to be transferred to a psychiatric hospital due to an acute psychosocial crisis that was not related to PR. Another patient had to quit PR because of disc prolapse. Another patient terminated study participation prematurely because he withdrew his consent for further study participation. All other 105 patients participated in at least $90 \%$ of the physicianprescribed therapies without related side effects.

\section{Prediction of successful PR concerning the primary outcome of dyspnea}

We did not find significant regression models predicting the reductions in one or both of the primary outcome measures. Further correlation analyses indicated that there were no statistically significant intercorrelations between the reductions in the primary outcome measures (exertional dyspnea NRS and mMRC) and sex $(p=0.153$ and $p=0.144)$, age $(p=0.758$ and $p=954)$, $\mathrm{BMI}(p=0.818$ and $p=0.585)$, duration of hospitalization $(p=0.506$ and $p=0.885)$, duration of intensive-care treatment $(p=0.912$ and $p=0.641)$, duration of ventilation $(p=0.539$ and $p=0.090)$, or the duration between the acute phase and the beginning of $\operatorname{PR}(p=0.149$ and $p=0.751)$. As displayed in Table 3 , the decrease in exertional dyspnea was also not correlated with an improvement in the $6 \mathrm{MWD}$ or improvements in parameters of the lung function tests or the laboratory measures.

\section{Discussion}

This prospective observational study is the second peer reviewed published study conducted in Germany and, to date, the largest to analyze the safety, feasibility, and efficacy of a three-week inpatient PR treatment in patients after COVID-19. All patients admitted due to COVID-19 between April 28 th, 2020 and January 08th, 2021 were asked to participate in the study, of whom $90 \%$ participated. The baseline values of our patient population are broadly comparable to those of Glöckl et al. [43]. Thus, our patient cohort seems to be representative of post-COVID-19 PR patients in Germany.

Almost all assessed outcomes improved significantly in the whole group at the end of PR, with moderate to large effect sizes. While negative outcomes, such as dyspnea, depression, and fatigue, were alleviated, measurements of performance and wellbeing, such as lung capacity, exercise capacity, and QoL, increased. We detected large effect sizes (Cohen's $d[42] d>0.8$ ) for the intensity of exertional dyspnea (primary outcome), physical submaximal exercise capacity, QoL, fatigue, and depression. Regarding effect size, the two strongest improvements were found for the 6MWD $(d=1.36)$ and the VAS scale of the EQ-5D-5L questionnaire ( $d=1.088)$, indicating that at the end of PR, patients achieved a significant improvement in physical performance and QoL.

Furthermore, moderate effects $(0.5 \leq d<0.8)$ were found for resting dyspnea and dyspnea in daily life in the last 7 days as measured by the mMRC. Cohen's $d$ $>0.4$ was found not only for important objectively measured lung function parameters (VC, FEV1, PaO2) but also for a decrease in anxiety. Significant but 
rather small effect sizes $(0.2<d<0.4)$ were found for cough, sputum, pain, other lung function parameters (TLC, TLCO, PI max), and results of the laboratory blood tests (D-dimers, CRP).

There was a high burden of comorbidities. In only 2 cases, no secondary diagnoses were mentioned. This figure is higher than what is reported in the literature for the overall group of COVID-19 patients [44, 45] but is in line with cross-sectional studies in post-COVID-19 rehabilitation settings [46-48]. This difference could be explained by the fact that pre-existing comorbidities negatively influence the course of COVID-19, and patients with a more severe course are more likely to undergo post-COVID-19 rehabilitation. The most frequently mentioned comorbidities were cardiovascular diseases and obesity. This finding is in line with existing data [45]. In addition, orthopedic and neuromuscular comorbidities were frequent, and some ICU-associated, psychological, and internal diseases were observed. These comorbidities require the multimodal, multiprofessional, and interdisciplinary approach of PR. Previous studies regarding rehabilitation following COVID-19 have reported similar frequencies of comorbidities [48-51]. This finding supports the necessity of a multimodal and interdisciplinary therapeutic approach for post-COVID-19 rehabilitation.

\section{Effectiveness of PR}

Comparing the three subgroups, for some outcomes, stronger effects were found in groups A and B. For example, exertional dyspnea improved in groups $\mathrm{A}$ and B, with large effect sizes $(d=0.922$ and $d=0.845$, respectively). Group C (mild after interval) also improved significantly but with a rather low to moderate effect size $(d=0.485)$. Similar trends were found for some lung function parameters (VC, FEV1, TLC, PaO2). Furthermore, patients in group $C$ rated both the efficacy of the PR and the personal improvement lower than the other two subgroups. In conclusion, these results indicate that patients with severe forms of COVID-19 show greater improvements over the course of PR, especially if the beginning of treatment occurs soon after the acute phase. These results are in line with the findings obtained by Al Chikhanie et al. [52]. However, it is important to emphasize that significant improvements were also found in group $\mathrm{C}$, with some strong (6MWD, EQ-5D-5L-VAS) and moderate effect sizes (PHQ-9). Cohen's $d$ values $>0.4$ were also found in group $\mathrm{C}$ regarding the intensity of exertional dyspnea, dyspnea in daily life, and impairments in QoL. This indicates that despite a lower effect size, patients with an initially mild course of the disease still benefit from PR even after a long duration, as described by Glöckl et al.

\section{Primary outcome: dyspnea}

Exertional dyspnea was mentioned as the "most important symptom" by the majority of patients in all three groups (groups A, B, and C in $65 \%, 50 \%$, and $40 \%$, respectively). Regarding the intensity of exertional dyspnea, as measured by NRS, all 3 subgroups benefited significantly, with large (groups A and B) or moderate (group C) effect sizes. These improvements were in the same range as those in our recent study on PR for asthma patients [53]. However, the decrease in NRS scores was not associated with improvements in lung function or 6MWD, but there were significant correlations with the reduction in fatigue and especially anxiety $(r=0.348)$. Possibly, the self-confidence in one's own performance regained through training during PR and the resulting reduction in the fear of exertion contributes to a reduction in exertional dyspnea.

Although most lung function parameters (VC, TLC, FEV1, TLCO, PaO2) in group C were normal on average and were higher than those in groups $\mathrm{A}$ and $\mathrm{B}$, group $\mathrm{C}$ scored their resting dyspnea higher at the start of PR and still scored higher at the end of PR. The more pronounced fatigue and the tendentially lower training effects of group $\mathrm{C}$ might have had an influence in this regard.

Regarding the mMRC dyspnea scale, which records shortness of breath in everyday life in the last week, there was a significant improvement, with a moderate effect size. This is worth noting because the mMRC dyspnea scale is considered to have low sensitivity to change. The improvement in the mMRC dyspnea scale is descriptively above the mean improvement seen in a large study on PR for COPD patients [53]. However, similar to the reduction in NRS values, the decrease in mMRC scores was not correlated with an increase in lung function but with a decrease in fatigue and anxiety.

Very few post-COVID-19 rehabilitation studies have reported results on dyspnea scores. A dyspnea assessment using the mMRC dyspnea scale was reported for a subgroup in the study by Glöckl et al.[43] $(\mathrm{N}=26$ patients after severe to critical COVID-19). In this study, the mean mMRC at the start of PR was 2 . This finding is comparable to the findings in groups $A$ and $B$, as is the change after PR. Furthermore, the decrease in patients reporting an $m M R C$ score of $\geq 2$ is comparable (77-54\% in Glöckl et al.; $79-54 \%$ in our study). In the early rehabilitation group of Curci et al. [48] ( $\mathrm{N}=41$ still severely impaired patients, directly transferred from an ICU to the COVID-19 rehabilitation unit of "Policlinico San Marco" Hospital, Zingonia, Bergamo, Italy), which is comparable to our population only to a very limited extent, $90.2 \%$ of patients documented an mMRC level of 4 . All of the patients improved by at least 1 level; the corresponding figures in our group A were $43.9 \%$ at rehabilitation onset, of whom $83.3 \%$ improved by at least 1 level at discharge. Thus, the changes in mMRC values we detected over the course of PR are comparable to those detected in other studies.

\section{Physical capacity, other clinical symptoms, and other objectively measured parameters}

A reduced exercise capacity compared to that in the healthy reference population was observed in all patients at $T_{1}$. The largest deviations were found in group A. Such values, however, should be interpreted with caution, since there are various, sometimes conflicting, normative value calculations for the 6MWD of healthy individuals [30,54-56]. With certain caveats, a walking distance of approximately 550 meters for women and 600 meters for men can be considered normal for our population. The observed 6MWD values are in the range of COPD rehabilitants in Germany [53,57] and are slightly above the values for patients suffering from interstitial lung diseases [58]. However, the values may vary with the time interval from the acute phase of the disease and decrease with disease severity, as was shown for post-COVID-19 rehabilitants by Glöckl et al.[43]. This may explain the fact that the 6MWDs of group B and group $C$ were higher than those reported for COVID-19 patients directly after an acute infection [46, 47, 59]. In all subgroups, the 6MWD improved, with large effect sizes, in groups A, B and C by $131 \mathrm{~m}$ (34.8\%), $96 \mathrm{~m}$ (20.9\%), and $74 \mathrm{~m}(15.4 \%)$, respectively. The changes in groups A and B are descriptively greater than the improvements achieved in our own PR studies concerning COPD or asthma patients [57,60]. The improvement in patients in group C, who started PR a mean of 143 days after the acute phase and whose pulmonary function parameters were normal except for a reduced PImax value, was well above the

Page $15 / 22$ 
MCID of patients with COPD [61-63] or idiopathic pulmonary fibrosis [64]. There were no significant correlations between the improvement in the 6MWD and lung function parameters except for a correlation with the improvement in PImax. Thus, we assume that the improved exercise capacity might not be a consequence of improved lung function but rather the result of adaptive mechanisms of the cardiovascular system and the musculature due to exercise training.

\section{Lung function tests}

The lung function pattern, which was more restrictive at baseline, improved in the overall group with a moderate effect size, with significant improvements in groups A and B. Similar lung function improvements were also seen in some other post-COVID-19 PR studies [43, 46, 52]. Maximal inspiratory pressure also improved in the total group, with a small effect size, with the most severe limitation and the smallest nonsignificant improvement seen in group $\mathrm{C}$ at $\mathrm{T}_{1}$.

However, the changes in VC, TLC, and FEV1 did not correlate with improvements in exertional dyspnea or physical capacity.

\section{Quality of life, fatigue, depression, and anxiety}

In line with previous research [7, 10], we detected not only physical but also mental impairments and subjectively experienced impairments in health-related QoL in the sample at $T_{1}$. In all subgroups, patients reported impairments in health-related QoL and symptoms of fatigue, depression, and anxiety. Over the course of PR, these parameters improved significantly, with mostly large effect sizes. Patients reported lower levels of impairment in health-related QoL and a decrease in fatigue, depression, and anxiety. In comparison to our own results for depression and anxiety in rehabilitants with asthma or COPD at the beginning and at the end of PR $[60,65]$, the results can be considered comparable. Interestingly, both measures of QoL were significantly correlated with a reduction in fatigue. Furthermore, the increase in the EQ-5D-5L-VAS showed a moderate correlation with the reduction in anxiety. However, there were no significant correlations between either of the two measures of QoL and the decrease in depression. Considering these numbers and the fact that one-third of the patients reported either sadness regarding their own health status or faintness/lack of energy as their most burdening symptom at $T_{1}$, we assume that fatigue and anxiety are of particular importance in the subjectively experienced burden of disease regarding COVID-19. Noticeably, improvements in fatigue, anxiety, and QoL were the only variables that showed statistically significant correlations with reductions in exertional dyspnea. Even though these results align with each other, it is currently hard to fully interpret the findings because of a lack of studies that examine the role of anxiety and/or fatigue in PR following COVID-19. Therefore, we highly recommend a closer examination in this regard to further explain our findings.

Regarding the overall subjectively experienced current health status, our data revealed a significant improvement, with large effect sizes for all groups. Threequarters of the patients achieved an improvement in the VAS score above the MCID of 8 points.

Given the significant reductions in depression and particularly anxiety in all the subgroups, we endorse the conclusion of Demeco et al. [66], who recommended post-COVID-19 rehabilitation programs not only for physical reasons but also for psychological reasons.

Regarding fatigue, we found a significant difference between group $\mathrm{C}$ and groups $\mathrm{A}$ and $\mathrm{B}$. Group $\mathrm{C}$ reported the highest values of fatigue at $\mathrm{T}_{1}$ and achieved no significant changes over the course of PR. These results suggest that patients with initially rather mild courses of disease and leading fatigue symptoms may need more specific therapeutic approaches for the treatment of persisting fatigue symptoms. It is, however, important to point out the small sample size of 15 patients with fatigue scores for both measurements. Therefore, these results must be interpreted with adequate caution.

Safety and feasibility of PR in patients after COVID-19.

The rehabilitation program proved to be safe; in particular, no complications occurred, and all but two patients were able to complete PR. Moreover, the program proved to be feasible, since all patients were able to perform more than $90 \%$ of the prescribed therapies.

\section{Limitations}

There are some limitations that we must point out. First and foremost, the data were from an observational study rather than a randomized controlled trial. Therefore, we cannot state with certainty to what extent rehabilitation caused the improvements. Factors such as the natural course of recovery or regression toward the mean may also have had an influence on the data. Consequently, our results should be interpreted with caution. Furthermore, because of the sample size, some effect sizes may not be precisely estimated. This was most prominently observable in the analyses of group C, which only included 21 patients. Therefore, the analyses of this group in particular should be generalized with caution.

It is important to remark that most of the patients assigned to the clinic can be characterized as having an interest in PR and being compliant with treatment recommendations. Furthermore, patients with a positive prognosis for rehabilitation are in general more likely to be admitted to PR. Therefore, the results cannot be easily extrapolated to the total group of all post-COVID-19 patients.

The study relies on data from one clinic only. Although another recently published study of German PR with a comparable patient population found comparable results[43] further research is needed to validate our findings. Considering the aforementioned limitations, randomized trials are needed.

\section{Conclusions}

In summary, PR seems to be safe, feasible, and effective in patients after acute COVID-19, improving clinically relevant outcomes. This was true for all three groups analyzed, with a trend toward greater efficacy after 'severe courses' of COVID-19 and an earlier start of rehabilitation after the acute phase of the disease. However, it must be emphasized that significant and clinically relevant effects were also seen after longer intervals of latency following milder courses. Therefore, adequate PR should be recommended for all post-COVID-19 patients who remain symptomatic. 


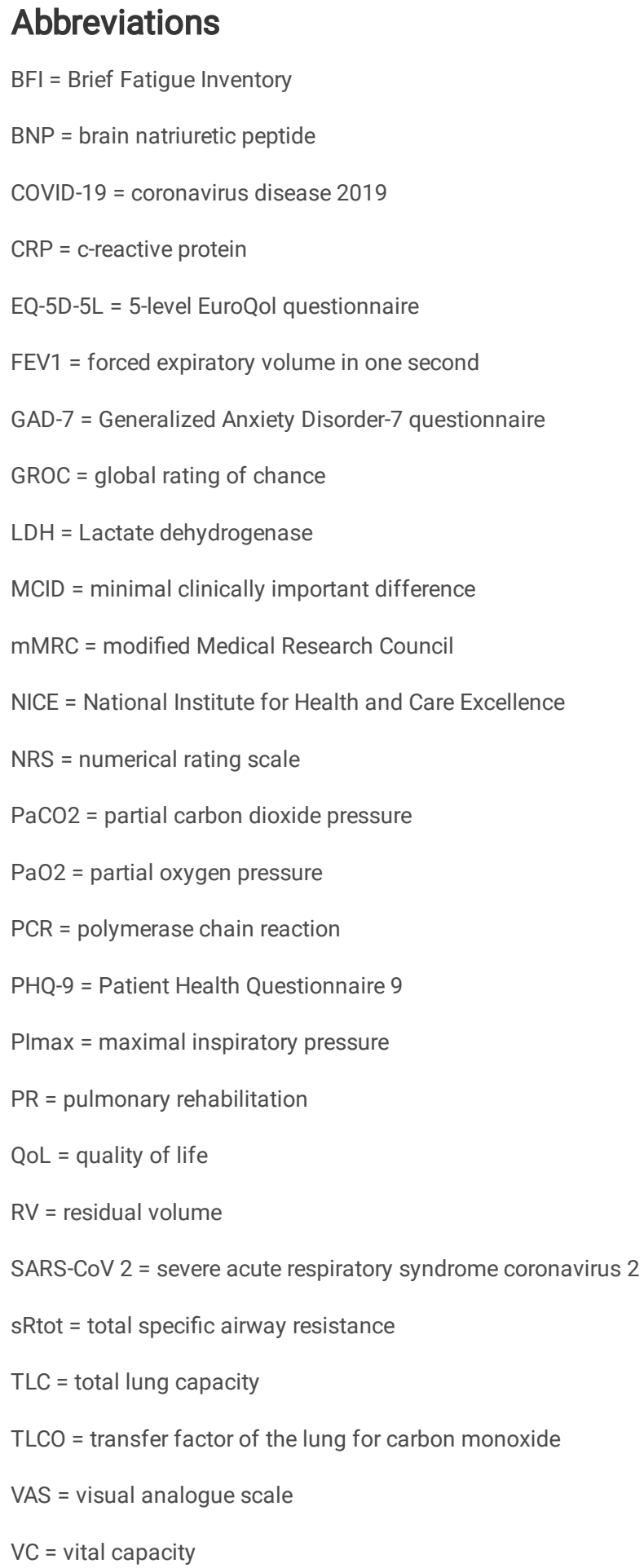

\section{Declarations}

\section{Ethics approval and consent to participate}

The study protocol was approved by the ethics committee of the medical faculty of the Ludwig-Maximilians-University Munich, Germany (No. 20-326) and is registered in the German register of clinical studies (DRKS00023180). Participation in the study was voluntary, and all the patients were informed that neither their refusal to participate nor their later withdrawal from the study would have any consequences whatsoever, particularly regarding therapy and aftercare. All participants provided written informed consent.

\section{Consent for publication}

Not applicable. 


\section{Availability of data and materials}

The raw data supporting the conclusions of this article will be made available by the authors, without undue reservation.

\section{Competing interests}

MH, ML, SM, GS, KJ, and KS are employees of the Bad Reichenhall Clinic. DN and MS declare no conflict of interest.

\section{Funding}

The study was carried out with the Bad Reichenhall Clinic's regular financial resources without any additional external funding.

\section{Authors' contributions}

The study was designed by MH, ML, KS, DW, and MS with assistance from GS and KJ. MH, ML, KS, GS, and KJ directed the study and supervised the data collection in the clinic. Data were collected by SM, ML, and MH. MS, MH, and ML analyzed the data and discussed the results. The first draft of the manuscript was written by $\mathrm{MH}, \mathrm{KS}, \mathrm{ML}$, and $\mathrm{MS}$. All authors contributed and agreed to the final manuscript.

\section{Acknowledgments}

We would like to thank all patients at the Bad Reichenhall Clinic who agreed to participate in the study. We would also like to thank the ward doctors who informed the patients and included them in the study, as well as the nursing staff for their support in collecting the study data. We would also like to thank Ms. Velicic, Ms. Seidl, and Ms. Köberl for their logistical support and Ms. Hinterseer and Mr. Botosch for their help with data processing.

\section{References}

1. Buitrago-Garcia D, Egli-Gany D, Counotte MJ, Hossmann S, Imeri H, Ipekci AM, Salanti G, Low N: Occurrence and transmission potential of asymptomatic and presymptomatic SARS-CoV-2 infections: A living systematic review and meta-analysis. PLoS Med 2020, 17(9):e1003346.

2. Schilling J, Lehfeld A-S, Schumacher D, Diercke M, Buda S, Haas W, Group RC-S: Krankheitsschwere der ersten COVID-19-Welle in Deutschland basierend auf den Meldungen gemäß Infektionsschutzgesetz. Journal of Health Monitoring 2020(S11):1-20.

3. Docherty AB, Harrison EM, Green CA, Hardwick HE, Pius R, Norman L, Holden KA, Read JM, Dondelinger F, Carson G et al: Features of 20133 UK patients in hospital with covid-19 using the ISARIC WHO Clinical Characterisation Protocol: prospective observational cohort study. BMJ 2020, 369:m1985.

4. Mazza MG, De Lorenzo R, Conte C, Poletti S, Vai B, Bollettini I, Melloni EMT, Furlan R, Ciceri F, Rovere-Querini P et al: Anxiety and depression in CoVID-19 survivors: Role of inflammatory and clinical predictors. Brain Behav Immun 2020, 89:594-600.

5. Bennett S, Tafuro J, Mayer J, Darlington D, Wong CW, Muntean EA, Wong N, Mallen C, Kwok CS: Clinical features and outcomes of adults with coronavirus disease 2019: A systematic review and pooled analysis of the literature. Int J Clin Pract 2020:e13725.

6. Huang C, Huang L, Wang Y, Li X, Ren L, Gu X, Kang L, Guo L, Liu M, Zhou X et al: 6-month consequences of CoVID-19 in patients discharged from hospital: a cohort study. Lancet 2021, 397(10270):220-232.

7. Halpin SJ, Mclvor C, Whyatt G, Adams A, Harvey O, McLean L, Walshaw C, Kemp S, Corrado J, Singh R et al: Postdischarge symptoms and rehabilitation needs in survivors of COVID-19 infection: A cross-sectional evaluation. J Med Virol 2020.

8. Carfi A, Bernabei R, Landi F, Gemelli Against C-P-ACSG: Persistent Symptoms in Patients After Acute COVID-19. JAMA 2020.

9. Mandal S, Barnett J, Brill SE, Brown JS, Denneny EK, Hare SS, Heightman M, Hillman TE, Jacob J, Jarvis HC et al: 'Long-CoVID': a cross-sectional study of persisting symptoms, biomarker and imaging abnormalities following hospitalisation for COVID-19. Thorax 2020.

10. Nalbandian A, Sehgal K, Gupta A, Madhavan MV, McGroder C, Stevens JS, Cook JR, Nordvig AS, Shalev D, Sehrawat TS et al: Post-acute CoVID-19 syndrome. Nat Med 2021, 27(4):601-615.

11. Armange L, Bénézit F, Picard L, Pronier C, Guillot S, Lentz P-A, Carré F, Tattevin P, Revest M: Prevalence and characteristics of persistent symptoms after non-severe COVID-19: a prospective cohort study. European Journal of Clinical Microbiology \& Infectious Diseases 2021.

12. Ordinola Navarro A, Cervantes-Bojalil J, Cobos Quevedo OJ, Avila Martinez A, Hernandez-Jimenez CA, Perez Alvarez E, Gonzalez Gil A, Peralta Amaro AL, Vera-Lastra O, Lopez Luis BA: Decreased quality of life and spirometric alterations even after mild-moderate COVID-19. Respir Med 2021, 181:106391.

13. Stavem K, Ghanima W, Olsen MK, Gilboe HM, Einvik G: Prevalence and Determinants of Fatigue after COVID-19 in Non-Hospitalized Subjects: A Population-Based Study. Int J Environ Res Public Health 2021, 18(4).

14. Nehme M, Braillard O, Alcoba G, Aebischer Perone S, Courvoisier D, Chappuis F, Guessous I: COVID-19 Symptoms: Longitudinal Evolution and Persistence in Outpatient Settings. Ann Intern Med 2020.

15. COVID-19 rapid guideline: managing the long-term effects of COVID-19. In.: (NICE) National Institute for Health and Care Excellence (UK), 2020; https://www.nice.org.uk/guidance/ng188\#: :text=This\%20guideline\%20covers\%20identifying\%2C\%20assessing,start\%20of\%20acute\%20COVID\%2D19.

16. Spruit MA, Holland AE, Singh SJ, Tonia T, Wilson KC, Troosters T: COVID-19: Interim Guidance on Rehabilitation in the Hospital and Post-Hospital Phase from a European Respiratory Society and American Thoracic Society-coordinated International Task Force. Eur Respir J 2020. 
17. Glockl R, Buhr-Schinner H, Koczulla AR, Schipmann R, Schultz K, Spielmanns M, Stenzel N, Dewey S: [Recommendations from the German Respiratory Society for Pulmonary Rehabilitation in Patients with COVID-19]. Pneumologie 2020, 74(8):496-504.

18. Vitacca M, Lazzeri M, Guffanti E, Frigerio P, D'Abrosca F, Gianola S, Carone M, Paneroni M, Ceriana P, Pasqua F et al: Italian suggestions for pulmonary rehabilitation in COVID-19 patients recovering from acute respiratory failure: results of a Delphi process. Monaldi Arch Chest Dis 2020, 90(2).

19. DE Sire A, Andrenelli E, Negrini F, Patrini M, Lazzarini SG, Ceravolo MG, International Multiprofessional Steering Committee of Cochrane Rehabilitation REHCA: Rehabilitation and COVID-19: a rapid living systematic review by Cochrane Rehabilitation Field updated as of December 31 st, 2020 and synthesis of the scientific literature of 2020. Eur J Phys Rehabil Med 2021.

20. Andrenelli E, Negrini F, de Sire A, Patrini M, Lazzarini SG, Ceravolo MG, International Multiprofessional Steering Committee of Cochrane Rehabilitation REHCa: Rehabilitation and COVID-19: update of the rapid living systematic review by Cochrane Rehabilitation Field as of February 28th, 2021. Eur J Phys Rehabil Med 2021.

21. Borg GA: Psychophysical bases of perceived exertion. Med Sci Sports Exerc 1982, 14(5):377-381.

22. Holland AE, Spruit MA, Troosters T, Puhan MA, Pepin V, Saey D, McCormack MC, Carlin BW, Sciurba FC, Pitta F et al: An official European Respiratory Society/American Thoracic Society technical standard: field walking tests in chronic respiratory disease. Eur Respir J 2014, 44(6):1428-1446.

23. Gift AG, Narsavage G: Validity of the numeric rating scale as a measure of dyspnea. Am J Crit Care 1998, 7(3):200-204.

24. Oxberry SG, Bland JM, Clark AL, Cleland JG, Johnson MJ: Minimally clinically important difference in chronic breathlessness: every little helps. Am Heart $J$ 2012, 164(2):229-235.

25. Salaffi F, Stancati A, Silvestri CA, Ciapetti A, Grassi W: Minimal clinically important changes in chronic musculoskeletal pain intensity measured on a numerical rating scale. Eur J Pain 2004, 8(4):283-291.

26. Bestall JC, Paul EA, Garrod R, Garnham R, Jones PW, Wedzicha JA: Usefulness of the Medical Research Council (MRC) dyspnoea scale as a measure of disability in patients with chronic obstructive pulmonary disease. Thorax 1999, 54(7):581-586.

27. Santus P, Tursi F, Croce G, Di Simone C, Frassanito F, Gaboardi P, Airoldi A, Pecis M, Negretto G, Radovanovic D: Changes in quality of life and dyspnoea after hospitalization in COVID-19 patients discharged at home. Multidiscip Respir Med 2020, 15(1):713.

28. Mahler DA, Wells CK: Evaluation of clinical methods for rating dyspnea. Chest 1988, 93(3):580-586.

29. Crisafulli E, Clini EM: Measures of dyspnea in pulmonary rehabilitation. Multidiscip Respir Med 2010, 5(3):202-210.

30. Enright PL, Sherrill DL: Reference equations for the six-minute walk in healthy adults. Am J Respir Crit Care Med 1998, 158(5 Pt 1):1384-1387.

31. Criee CP, Baur X, Berdel D, Bosch D, Gappa M, Haidl P, Husemann K, Jorres RA, Kabitz HJ, Kardos P et al: [Standardization of spirometry: 2015 update. Published by German Atemwegsliga, German Respiratory Society and German Society of Occupational and Environmental Medicine]. Pneumologie 2015, 69(3):147-164

32. Criee CP, Sorichter S, Smith HJ, Kardos P, Merget R, Heise D, Berdel D, Kohler D, Magnussen H, Marek W et al: Body plethysmography-its principles and clinical use. Respir Med 2011, 105(7):959-971.

33. Radbruch L, Sabatowski R, Elsner F, Everts J, Mendoza T, Cleeland C: Validation of the German version of the brief fatigue inventory. J Pain Symptom Manage 2003, 25(5):449-458.

34. Mendoza TR, Wang XS, Cleeland CS, Morrissey M, Johnson BA, Wendt JK, Huber SL: The rapid assessment of fatigue severity in cancer patients: use of the Brief Fatigue Inventory. Cancer 1999, 85(5):1186-1196.

35. Oemar M, Janssen B: EQ-5D-5L User Guide2013; (Version 2.0, October 2013)..

36. Zanini A, Aiello M, Adamo D, Casale S, Cherubino F, Della Patrona S, Raimondi E, Zampogna E, Chetta A, Spanevello A: Estimation of minimal clinically important difference in EQ-5D visual analog scale score after pulmonary rehabilitation in subjects with COPD. Respir Care 2015, 60(1):88-95.

37. Kroenke K, Spitzer RL, Williams JB: The PHQ-9: validity of a brief depression severity measure. J Gen Intern Med 2001, 16(9):606-613.

38. Spitzer RL, Kroenke K, Williams JB, Lowe B: A brief measure for assessing generalized anxiety disorder: the GAD-7. Arch Intern Med 2006, 166(10):10921097.

39. Gräfe K, Zipfel S, Herzog W, Löwe B: Screening psychischer Störungen mit dem "Gesundheitsfragebogen für Patienten (PHQ-D)". Diagnostica 2004, 50:171-181.

40. Kamper SJ, Maher CG, Mackay G: Global rating of change scales: a review of strengths and weaknesses and considerations for design. J Man Manip Ther 2009, 17(3):163-170.

41. Alma H, de Jong C, Jelusic D, Wittmann M, Schuler M, Flokstra-de Blok B, Kocks J, Schultz K, van der Molen T: Health status instruments for patients with COPD in pulmonary rehabilitation: defining a minimal clinically important difference. NPJ Prim Care Respir Med 2016, 26:16041.

42. Cohen J: Statistical power analysis for the behavioral sciences, 2nd edn. Hillsdale, N.J.: L. Erlbaum Associates; 1988.

43. Gloeckl R, Leitl D, Jarosch I, Schneeberger T, Nell C, Stenzel N, Vogelmeier C, Kenn K, Koczulla AR: Benefits of pulmonary rehabilitation in CoVID-19 - a prospective observational cohort study. ERJ Open Res, 2021, in press

44. Bajgain KT, Badal S, Bajgain BB, Santana MJ: Prevalence of comorbidities among individuals with COVID-19: A rapid review of current literature. Am J Infect Control 2021, 49(2):238-246.

45. Ng WH, Tipih T, Makoah NA, Vermeulen JG, Goedhals D, Sempa JB, Burt FJ, Taylor A, Mahalingam S: Comorbidities in SARS-CoV-2 Patients: a Systematic Review and Meta-Analysis. mBio 2021, 12(1).

46. Puchner B, Sahanic S, Kirchmair R, Pizzini A, Sonnweber B, E WO, A MU, Garimorth K, Dareb B, Ehling R et al: Beneficial effects of multi-disciplinary rehabilitation in post-acute COVID-19 - an observational cohort study. Eur J Phys Rehabil Med 2021.

Page $19 / 22$ 
47. Spielmanns M, Pekacka-Egli AM, Schoendorf S, Windisch W, Hermann M: Effects of a Comprehensive Pulmonary Rehabilitation in Severe Post-COVID-19 Patients. Int J Environ Res Public Health 2021, 18(5).

48. Curci C, Negrini F, Ferrillo M, Bergonzi R, Bonacci E, Camozzi DM, Ceravolo C, De Franceschi S, Guarnieri R, Moro P et al: Functional outcome after inpatient rehabilitation in post-intensive care unit COVID-19 patients: findings and clinical implications from a real-practice retrospective study. Eur $J$ Phys Rehabil Med 2021.

49. Bertolucci F, Sagliocco L, Tolaini M, Posteraro F: Comprehensive rehabilitation treatment for sub acute CoVID-19 patients: an observational study. Eur J Phys Rehabil Med 2021.

50. Wiertz CMH, Vints WAJ, Maas G, Rasquin SMC, van Horn YY, Dremmen MPM, Hemmen B, Verbunt JA: CoVID-19: patient characteristics in the first phase of post-intensive care rehabilitation. Arch Rehabil Res Clin Trans/ 2021:100108.

51. Hermann M, Pekacka-Egli AM, Witassek F, Baumgaertner R, Schoendorf S, Spielmanns M: Feasibility and Efficacy of Cardiopulmonary Rehabilitation After COVID-19. Am J Phys Med Rehabil 2020, 99(10):865-869.

52. Al Chikhanie Y, Veale D, Schoeffler M, Pepin JL, Verges S, Herengt F: Effectiveness of pulmonary rehabilitation in CoVID-19 respiratory failure patients post-ICU. Respir Physiol Neurobiol 2021, 287:103639.

53. Schultz K, Jelusic D, Wittmann M, Kramer B, Huber V, Fuchs S, Lehbert N, Wingart S, Stojanovic D, Gohl O et al: Inspiratory muscle training does not improve clinical outcomes in 3-week COPD rehabilitation: results from a randomised controlled trial. Eur Respir J 2018, 51(1).

54. Jay SJ: Reference equations for the six-minute walk in healthy adults. Am J Respir Crit Care Med 2000, 161(4 Pt 1):1396.

55. Casanova C, Celli BR, Barria P, Casas A, Cote C, de Torres JP, Jardim J, Lopez MV, Marin JM, Montes de Oca M et al: The 6-min walk distance in healthy subjects: reference standards from seven countries. Eur Respir J 2011, 37(1):150-156.

56. Oliveira MJ, Marcoa R, Moutinho J, Oliveira P, Ladeira I, Lima R, Guimaraes M: Reference equations for the 6-minute walk distance in healthy Portuguese subjects 18-70 years old. Pulmonology 2019, 25(2):83-89.

57. Greulich T, Koczulla AR, Nell C, Kehr K, Vogelmeier CF, Stojanovic D, Wittmann M, Schultz K: Effect of a Three-Week Inpatient Rehabilitation Program on 544 Consecutive Patients with Very Severe COPD: A Retrospective Analysis. Respiration 2015, 90(4):287-292.

58. Huppmann P, Sczepanski B, Boensch M, Winterkamp S, Schonheit-Kenn U, Neurohr C, Behr J, Kenn K: Effects of inpatient pulmonary rehabilitation in patients with interstitial lung disease. Eur Respir J 2013, 42(2):444-453.

59. Zampogna E, Paneroni M, Belli S, Aliani M, Gandolfo A, Visca D, Bellanti MT, Ambrosino N, Vitacca M: Pulmonary Rehabilitation in Patients Recovering from COVID-19. Respiration 2021, 100(5):416-422.

60. Schultz K, Wittmann M, Wagner R, Lehbert N, Schwarzkopf L, Szentes B, Nowak D, Faller H, Schuler M: In-patient pulmonary rehabilitation to improve asthma control-a randomized controlled study (EPRA, Effectiveness of Pulmonary Rehabilitation for Patients with Asthma). Dtsch Arztebl Int 2021, 118(3):23-30.

61. Puhan MA, Mador MJ, Held U, Goldstein R, Guyatt GH, Schunemann HJ: Interpretation of treatment changes in 6-minute walk distance in patients with COPD. Eur Respir J 2008, 32(3):637-643.

62. Puhan MA, Chandra D, Mosenifar Z, Ries A, Make B, Hansel NN, Wise RA, Sciurba F, National Emphysema Treatment Trial Research G: The minimal important difference of exercise tests in severe COPD. Eur Respir J 2011, 37(4):784-790.

63. Geidl W, Carl J, Schuler M, Mino E, Lehbert N, Wittmann M, Pfeifer K, Schultz K: Long-term benefits of adding a pedometer to pulmonary rehabilitation for COPD. The randomized controlled STAR trial. . Int J Chron Obstruct Pulmon Dis 2021, in press.

64. du Bois RM, Weycker D, Albera C, Bradford WZ, Costabel U, Kartashov A, Lancaster L, Noble PW, Sahn SA, Szwarcberg J et al: Six-minute-walk test in idiopathic pulmonary fibrosis: test validation and minimal clinically important difference. Am J Respir Crit Care Med 2011, 183(9):1231-1237.

65. Schuler M, Strohmayer M, Muhlig S, Schwaighofer B, Wittmann M, Faller H, Schultz K: Assessment of depression before and after inpatient rehabilitation in COPD patients: Psychometric properties of the German version of the Patient Health Questionnaire (PHQ-9/PHQ-2). J Affect Disord 2018, 232:268-275.

66. Demeco A, Marotta N, Barletta M, Pino I, Marinaro C, Petraroli A, Moggio L, Ammendolia A: Rehabilitation of patients post-CoVID-19 infection: a literature review. J Int Med Res 2020, 48(8):300060520948382.

\section{Figures}




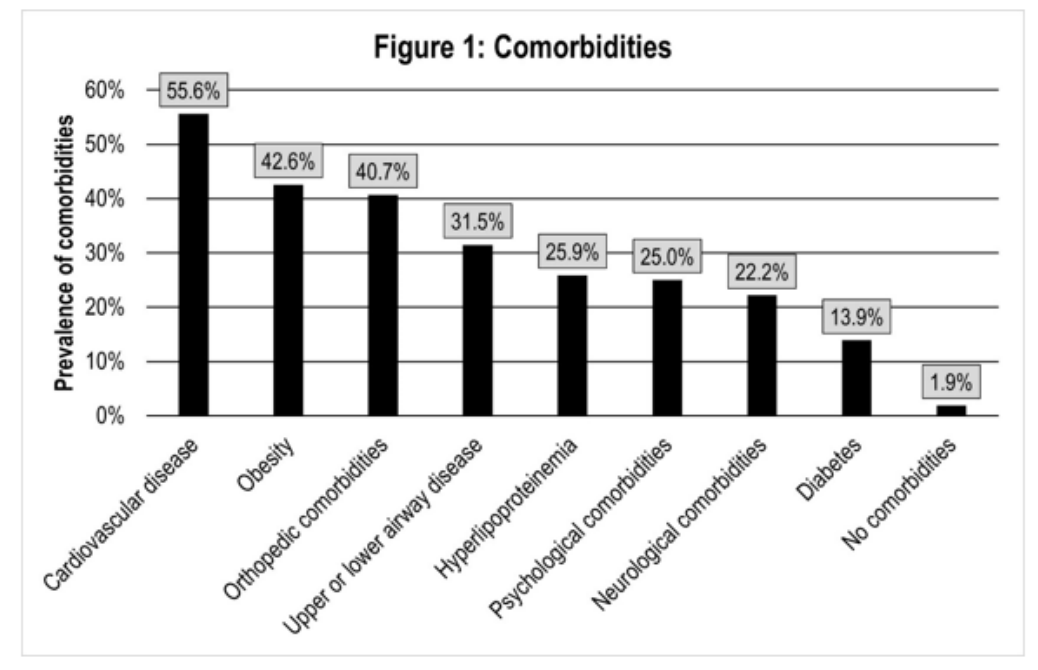

Figure 1

Comorbidities

\begin{tabular}{|c|c|c|c|c|c|c|c|c|c|c|c|c|c|}
\hline & NRS1 & & & $\bullet$ & $\longrightarrow$ & & & & & & \multicolumn{3}{|c|}{$0.41 \pm 0.23$} \\
\hline & NRS2 & & & & & & & & & & \multicolumn{3}{|c|}{$0.82 \pm 0.19$} \\
\hline & mMRC & & & & $\bullet$ & & & & & & \multicolumn{3}{|c|}{$0.64 \pm 0.23$} \\
\hline & 6MWD & & & & & & & - & & & \multicolumn{3}{|c|}{$1.36 \pm 0.27$} \\
\hline & vc & & & $\longrightarrow$ & 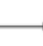 & & & & & & \multicolumn{3}{|c|}{$0.47 \pm 0.2$} \\
\hline & TLC & & & $\bullet$ & & & & & & & \multicolumn{3}{|c|}{$0.36 \pm 0.2$} \\
\hline & FEV1 & & & 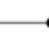 & 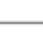 & & & & & & \multicolumn{3}{|c|}{$0.49 \pm 0.21$} \\
\hline & $\mathrm{PaO} 2$ & & & $\longmapsto$ & $\bullet$ & & & & & & \multicolumn{3}{|c|}{$0.55 \pm 0.21$} \\
\hline & TLCO & & & $\longmapsto$ & $\bullet$ & & & & & & \multicolumn{3}{|c|}{$0.58 \pm 0.21$} \\
\hline & Pimax & & & • & & & & & & & \multicolumn{3}{|c|}{$0.36 \pm 0.2$} \\
\hline & D-dimers & & & $\bullet$ & & & & & & & \multicolumn{3}{|c|}{$0.43 \pm 0.24$} \\
\hline & CRP & & & $\bullet$ & & & & & & & \multicolumn{3}{|c|}{$0.41 \pm 0.24$} \\
\hline & EQ-5D-5L & & & & & & & & & & \multicolumn{3}{|c|}{$0.95 \pm 0.26$} \\
\hline & VAS & & & & & & & & $\longrightarrow$ & & \multicolumn{3}{|c|}{$1.09 \pm 0.35$} \\
\hline & $\mathrm{BFI}$ & & & & & & & $\longrightarrow$ & & & \multicolumn{3}{|c|}{$0.99 \pm 0.26$} \\
\hline & PHQ-9 & & & & & & & $\bullet$ & $\longrightarrow$ & & \multicolumn{3}{|c|}{$1.18 \pm 0.29$} \\
\hline & GAD-7 & & & & & 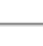 & & & & & \multicolumn{3}{|c|}{$0.66 \pm 0.24$} \\
\hline-0.8 & $\begin{array}{ccc}-0.6 & -0.4 & -0.2\end{array}$ & 0 & 0.2 & 0.4 & 0.6 & 0.8 & 1 & 1.2 & 1.4 & 1.6 & 1.8 & 2 & 2.2 \\
\hline
\end{tabular}

\section{Figure 2}

Changes in clinically relevant outcomes over the course of the rehabilitation (absolute value of Cohen's d and 95\% confidence interval) Notes: 6MWD: 6-minute walking distance; BFI: Brief Fatigue Inventory; CRP: C-reactive protein; EQ-5D: 5-level EQ-5D questionnaire; FEV1: forced expiratory volume in one second: GAD7: Generalized Anxiety Disorder-7; mMRC: modified Medical Research Council; NRS1: Numeric Rating Scale for resting dyspnea; NRS2: Numeric Rating Scale for exertional dyspnea; PaO2: partial pressure of 02; PHQ-9: Patient Health Questionnaire 9; PImax: maximal inspiratory pressure; TLC: total lung capacity; TLCO: diffusion capacity of the lungs for carbon monoxide; VAS: visual analog scale; VC: vital capacity 


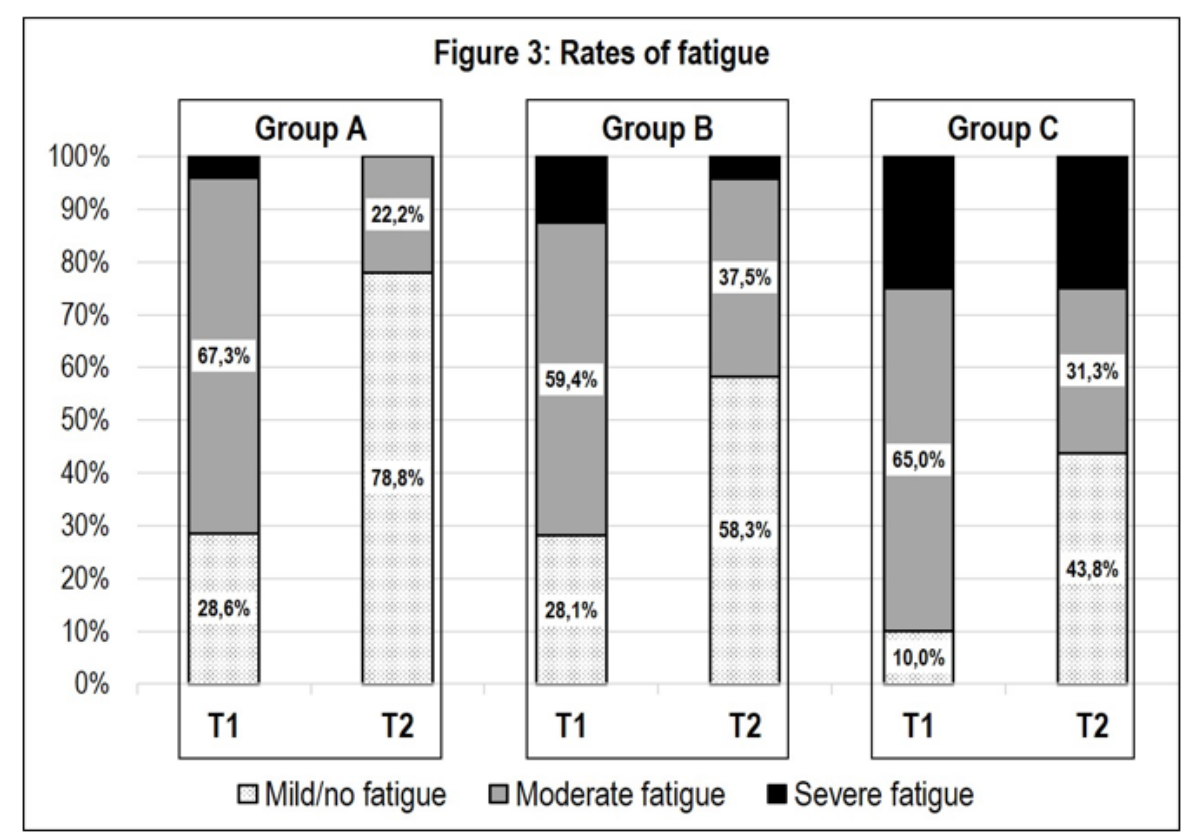

Figure 3

Rates of fatigue

\section{Supplementary Files}

This is a list of supplementary files associated with this preprint. Click to download.

- Supplement.docx 